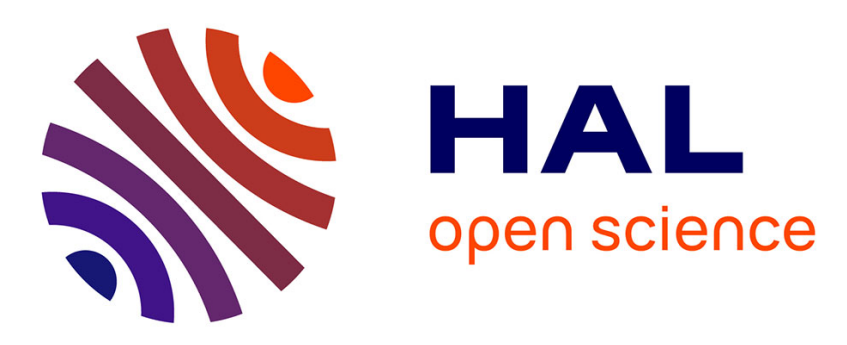

\title{
Pressure losses at moderate Reynolds numbers in diamond-shaped cylinders arrays: application to micro-regenerators
}

Arkadii Sochinskii, Damien Colombet, Manuel Medrano Muñoz, Frederic Ayela, Nicolas Luchier

\section{To cite this version:}

Arkadii Sochinskii, Damien Colombet, Manuel Medrano Muñoz, Frederic Ayela, Nicolas Luchier. Pressure losses at moderate Reynolds numbers in diamond-shaped cylinders arrays: application to micro-regenerators. Journal of Fluids Engineering, 2021. hal-03109853

\section{HAL Id: hal-03109853 https://hal.science/hal-03109853}

Submitted on 14 Jan 2021

HAL is a multi-disciplinary open access archive for the deposit and dissemination of scientific research documents, whether they are published or not. The documents may come from teaching and research institutions in France or abroad, or from public or private research centers.
L'archive ouverte pluridisciplinaire HAL, est destinée au dépôt et à la diffusion de documents scientifiques de niveau recherche, publiés ou non, émanant des établissements d'enseignement et de recherche français ou étrangers, des laboratoires publics ou privés. 


\title{
Pressure losses at moderate Reynolds numbers in diamond-shaped cylinders arrays: application to micro-regenerators
}

\author{
A. Sochinskii \\ PhD Student \\ Univ. Grenoble Alpes \\ CNRS, Grenoble INP, LEGI \\ Grenoble, France, 38000 \\ present adress: \\ Université côte d'azur \\ M. Medrano Muñoz \\ Assistant professor \\ Univ. Grenoble Alpes \\ CEA, IRIG, DSBT \\ Grenoble, France, 38000
}

\author{
D. Colombet \\ Assistant professor \\ Univ. Grenoble Alpes \\ CNRS, Grenoble INP, LEGI \\ Grenoble, France, 38000
}

Email: damien.colombet@univ-grenoble-alpes.fr

\author{
F. Ayela \\ Professor \\ Univ. Grenoble Alpes \\ CNRS, Grenoble INP, LEGI \\ Grenoble, France, 38000
}

\author{
N. Luchier \\ Senior researcher \\ CEA, Univ. Grenoble Alpes, IRIG, DSBT \\ Grenoble, France, 38000
}

\begin{abstract}
Cylinders with an elliptical, oblong, lenticular, sinus or diamond transveral shape are very interesting geometries for the design of compact heat exchangers. This work investigates the role of the porosity and of the apex angle of diamond-shaped cylinders networks on the pressure losses, at moderate Reynolds numbers, inside micro heat regenerators. The design of the geometry under test has been chosen so that the cross section of the flow remains almost constant along the path of the flow between cylinders. Experiments have been performed at $1 \leqslant R e \leqslant 30$ and a porosity range $0.40<\varepsilon<0.90$ for an apex angle of $\alpha=33^{\circ}$. Numerical simulations have been conducted using the same Reynolds and
\end{abstract}


porosity ranges but varying the apex angle $33^{\circ} \leqslant \alpha \leqslant 90^{\circ}$. Experimental measurements and dimensional analysis have shown that the friction factor can be affected by the porosity. $2 D$ numerical simulations confirmed that the friction factor increases with the porosity but also with the apex angle. An analysis at the scale of a channel flanked by adjacent cylinders has provided a original correlation able to describe easily the evolution of the Poiseuille number and the collective effects on the drag coefficient as a function of $\alpha$ and $\varepsilon$. Such a diamond-shaped design is found to induce much lower Poiseuille numbers than those expected from conventional stacked spheres, woven wires and circular cylinders arrays. The findings of this study can help for better understanding the optimization of low pressure drop regenerators and how to reduce associated hydraulic power.

\section{HIGHLIGHTS}

Poiseuille numbers are globally much lower than conventional arrays.

Poiseuille numbers increase with the porosity and with the apex angle.

The variation of the tortuosity explains Poiseuille variations with the porosity.

A new simple correlation is proposed to describe both Poiseuille numbers and drag.

\section{INTRODUCTION}

In the frame of process intensification, miniaturisation leads to the design of thermally efficient heat exchangers having the lowest energy consumption. The hydraulic power required to generate fluid flow is the product of the volumetric flow rate by the inlet/outlet pressure drop. For a given exchanger design it is thus important to not only evaluate heat transfer efficiency or thermallyoptimal arrangement, but also to characterize the corresponding pressure losses. , For the sake of simplicity, references in the literature are mostly related to cylinder shaped arrays also called 'tube bundle' or 'tube bank' [1, 2, 3, 4, 5, 6].

By designing compact cylinders array heat exchangers, the main solution to enhance heat flux per volume unit is to increase the total exchange area. This is usually achieved either by decreasing the cylinders size and increasing their number per volume unit, or by changing the 
cylinders aspect ratio and their shape. The main shapes studied for heat exchangers with noncircular cylinders arrays are elliptical or oblong [7, 8, 9], rectangle or square $[10,11,12,13,14]$, lenticular [15] but also, in more recent studies, sinus and diamond [16, 17, 18, 19, 20].

For in-line or staggered arrangement of square or diamond-shaped cylinders arrays with an apex angle of $\alpha=90^{\circ}$, heat transfer or pressure drop were characterized by Chyu et al. [17], Tanda [18] or Jeng [19]. Chyu et al. [17] found experimentally that staggered matrix of squared shaped cylinders exhibits a higher transfer rate than a staggered diamond shaped array, which exhibits an higher transfer rate than a staggered circular cylinders array. Globally staggered arrangements are found to generate higher heat transfer rates and pressure losses than in-line arrangements. The transfer was studied using a naphthalen sublimation technique. In an air flow through diamondshaped cylinders arrays, Tanda [18] has quantified in 2D mass transfer rate and temperature distribution for staggered and in-line arrangement using liquid crystal thermography and image processing. According to the authors, at the same hydraulic powers, diamond-shaped cylinders arrays can be up to 1.65 more efficient than an empty channel. Flow and heat transfer in a compact diamond-shaped cylinder exchanger was also studied experimentally by Rasouli et al. [21] using liquid nitrogen for thermal management applications in cryo-adsorbent hydrogen storage systems. The authors observed an increase of the friction factor with the Reynolds number above $R e>580-650$, because of flow flapping behind the cylinders. The thermal efficiency was also investigated experimentally by Jeng [19] for the case of in-line array of diamond-shaped cylinders using a transient method for measuring the heat transfer coefficient. The author has concluded that for a given hydraulic power it is possible to find an optimal distance between cylinders to maximize heat transfer.

It is important to stress that, in the literature, usually named diamond-shaped cylinders arrays are in fact $45^{\circ}$ inclined square-shaped cylinders (i.e. diamond shape with an apex angle of $\alpha=$ $90^{\circ}$ ). The effect of varying the apex angle (or cylinder section aspect ratio) is not systematically considered.

Heat exchangers with a diamond shape having an apex angle equal or lower to $90^{\circ}$ were studied for electronic cooling applications in space industry [16, 22, 20]. Pressure drop coeffi- 
cients in such geometries with an apex angle of 45 or $90^{\circ}$ and a porosity of $65 \%$ were characterised experimentally and numerically by Sparrow \& Grannis [16] for the Reynolds number range $20<R e<2700$, in order to give correlations to assist dimensioning of an air/air heat exchanger for a space shuttle. That question was in the same time studied numerically in a companion paper of Grannis \& Sparrow [22] using a finite-element method. The authors have simulated a periodic domain to investigate the flow and the pressure drop in similar diamond-shaped cylinder staggered arrangements. For $\alpha=45^{\circ}$, the authors propose two correlations of the friction factor and suggest that the Poiseuille number is quite constant for the range $0.22 \leqslant \varepsilon \leqslant 0.70$ for an arrangement corresponding to an equilateral triangular pattern $\left(P_{O} \approx 108\right)$ and to a regular staggered arrangement $\left(P_{o} \approx 97\right)$. For $\alpha=90^{\circ}$ and same arrangements, the authors propose correlations considering a singular pressure drop per row of cylinders. In this reference, the authors admitted that it is very difficult to collapse results with $\alpha=45^{\circ}$ and $\alpha=90^{\circ}$ under a common description.

Later, diamond-shaped cylinder networks were also investigated by Vanapalli et al. [20] for their use as a micro-heat exchanger for the cryocooling of satellite electronic. For laminar flows at moderate Reynolds numbers $(50 \leqslant R e \leqslant 500)$ and apex angle of $\alpha=37.15^{\circ}$, [20] found experimentally that a diamond-shaped cylinder matrix generates a higher pressure drop than circular, square, elliptical or sinus shapes.

For a turbulent flow regime and downstream an array of diamond-shaped cylinders, the jet formation with a specific flapping instability has been observed at the outlet of a liquid flow emerging into air [23, 24]. For such a flow, by using numerical simulations and experimental measurements, Hirasawa et al. [25] proposed correlations to describe the pressure drop and heat transfer in an array of diamond-shaped cylinders with an apex angle of $\alpha=30^{\circ}$.

In micro-fluidics, the diamond-shaped cylinders arrays have also been investigated for liquid phase chromatography applications $[26,27,28]$. This geometry seems to offer better chromatographic performances than circular or ellipse cylinders arrays. Finally, porous diamond-shaped cylinders are also expected to be interesting for biological filtering processes [29]. As a result, various applications could benefit from the knowledge of the pressure drop in an array of diamondshaped cylinders. 
In the literature, the pressure losses in cylinders arrays depends on the compactness of the heat exchanger. This is usually quantified by considering the exchanger porosity that is the ratio between the volume occupied by the fluid to the total volume of the exchanger. Pressure losses in non compact heat exchangers with cylinders arrays at high and moderate porosity are presented as the sum of singular pressure losses controlled by the number of cylinder rows in the array, using the maximal velocity in the network for the calculation of the Reynolds number $[1,3,16,8,5]$. This approach can be justified for a dilute array of cylinders, where a row of cylinders can be considered as a singularity. For more compact exchanger, decreasing the porosity make the exchanger close to a porous media and pressure losses can be described as a linear pressure drop considering the friction of the flow on the walls. This approach consists in using the Darcy-Weisbach coefficient is typically the one employed for compact heat exchangers $[30,22,12,18,13,20,19,14]$ using a mean velocity for the Reynolds calculation. In addition, a large number of correlations exists to describe the pressure drop in cylinders arrays. Usually it is found from numerical or experimental works that the friction factor is not only controlled by the Reynolds number but also monitored by the lateral or longitudinal dimensionless distances and the arrangement (in-line or staggered [31]). Different comparative studies have pointed out the fact that available correlations in the literature are not able to predict experimental pressure drop accurately (see [32, 33] and [34]). As a matter of fact, even if the literature on that subject is large, the understanding of flow behaviour and associated pressure losses in cylinders arrays remains a question of interest. Moreover, to our knowledge, for diamond-shaped cylinder arrays, no available correlation able to predict pressure losses for a wide range of porosity and apex angle has been published.

The paper is organized as follows. First, the geometrical parameters of the exchanger under investigation and its micro-fabrication are presented. Then, the parameters used to characterize the flow are introduced. After that, the experimental investigations performed are detailed for diamond-shaped cylinders arrays with an apex angle of $\alpha=33^{\circ}$ and a porosity of $0.45 \leqslant \varepsilon \leqslant 0.78$. Finally, a numerical investigation is achieved for the same geometry and porosity range but with a larger range of apex angle $33^{\circ} \leqslant \alpha \leqslant 90^{\circ}$. In those two last sections, preliminary validation tests are performed and results are compared to the literature. 
The main novelity of this work is to extend the investigations of Sparrow \& Grannis $([16,22]$ which were limited to a single porosity $\left(\varepsilon=65 \%\right.$ and $\alpha=45$ or $\left.90^{\circ}\right)$ at a much larger range on the porosity $\varepsilon$ but also on the apex angle $\alpha$. The second asset of this work is to consider a specific staggered arrangement intended to maintain a constant flow cross section, that is known to be an important parameter to minimize pressure drops as shown previously by Ruhlich \& Quack [15] thanks to a numerical study on the optimisation of regenerators design. Indeed, if the flow cross section varies continuously and significantly, one important contribution to the pressure losses is the flow acceleration and deceleration along its path. In this work, a geometrical constraint is imposed to follow this principle and keep the flow cross section quite constant. The third advantage of this work is to propose to use three complementary approaches (experiments, numerical simulations, dimensional analysis) to bring new findings on that topic. Finally, compared to many other previous works using random arrays of spheres or wires as a regenerator, as already performed by a few research teams before (see for example [20]), the last originality of this study is to control perfectly the geometry of the regenerators thanks to the developpment of MEMS techniques.

\section{GEOMETRICAL PARAMETERS}

The samples used in our study consist of straight channels with a rectangular cross section and with micro structures inside, that act as a porous media. Figure 1 shows the layout and a picture of one of our sample. The width and the length of the main channel are the same for all the samples. The circular tanks at the ends are used to connect the inlet and outlet easily. In our experiments, the porous area consists of a micro-size solid cylinders matrix located in the center of the channel. These cylinders are diamond-shaped with an apex angle $\alpha$ equals to 33 degrees (see Fig, 1a). This angle corresponds to an aspect ratio of 0.30 that was identified in a previous work to be a potential compromise between efficient heat transfer and moderate pressure losses [15]. In the numerical investigations, the apex angle has been changed to estimate the influence of the apex angle on the pressure drop.

The present work focuses on the case of a periodic staggered arrangement of diamond shaped cylinders as presented in Fig. 2 with a width ' $a$ ' and length ' $b$ ' that depends on the apex angle 
$\alpha=2 \times \operatorname{atan}(a / b)$. The parameter $c=\sqrt{\left[(a / 2)^{2}+(b / 2)^{2}\right]}$ is the length of one of the cylinder face so that for one cylinder the wetted surface in contact with the fluid is $4 \mathrm{cH}$, where $H$ is the cylinder height. ' $e$ ' is the distance between two cylinders faces, but it can also be presented as the width of the channel formed between the diamond-shaped cylinders. $\Delta x$ stands for the distance between the front (resp. rear) apex angle and the inlet (resp. outlet) of the chosen periodic domain. $\Delta y$ corresponds to the distance between the top (resp. bottom) equator angle and the top (resp. bottom) side of the periodic domain. $L_{L}=b+2 \Delta x$ and $L_{T}=a+2 \Delta y$ are respectively the length and width of the periodic pattern.

As explained in the introduction, to minimize the singular pressure losses due to fluid acceleration/deceleration, heat exchanger geometries having a quasi constant cross section between matrix columns have been investigated in the present work. To fulfill this condition, the geometrical constraint $e=\Delta y$ has been imposed to the studied gemotries. From Fig. 2b, with this constraint, it can be shown that this imposes $\pi=2 \beta+\pi / 2+\theta$ and $\pi=\pi / 2+\theta+\alpha / 2$ (upper triangle) so that $\beta=\alpha / 4$ and then $\Delta x=e \tan (\alpha / 4)$. Consequently, the size of a periodic domain for such geometries is simply $L_{L}=b+2 e \tan (\alpha / 4)$ with $L_{T}=a+2 e$.

It is now possible to define the exchanger porosity (or volume fraction of fluid) as a function of the geometrical parameters as follows

$$
\varepsilon=V_{\text {fluid }} / V_{\text {total }}=1-\frac{V_{\text {cylinders }}}{V_{\text {total }}}=1-\frac{a b}{(b+2 e \tan (\alpha / 4))(a+2 e)}
$$

with $V_{\text {total }}=V_{\text {fluid }}+V_{\text {cylinders }}=L_{L} L_{T} H=(b+2 \Delta x)(a+2 \Delta y) H=(b+2 e \tan (\alpha / 4))(a+2 e) H$ and $V_{\text {cylinders }}=2(a b / 2) H=a b H$.

According to the porous media approach, the hydraulic diameter of the heat exchanger matrix is defined as follow 


$$
D_{h}=\frac{4 \varepsilon V_{\text {total }}}{A_{\text {cont }}+A_{\text {cont }}^{W}}
$$

where the wetted surface in contact with the column is $A_{\text {cont }}=8 \mathrm{cH}$. In our experiments the wetted surface in contact with the top an bottom channel walls is $A_{\text {cont }}^{W}=2 L_{L} L_{T} \varepsilon$ while for $2 \mathrm{D}$ simulations one has $A_{c o n t}^{W}=0$. In practice, experimental samples were designed by varying the porosity between 0.4 and 0.8 with $\alpha \approx 33^{\circ}$ for a given cylinder distance 'e' (10 - 20 - $\left.40 \mu \mathrm{m}\right)$.

For a fully 2D geometry, where $H$ is much larger than all other lengths as in numerical simulations $\left(A_{\text {cont }}>>A_{\text {cont }}^{W}\right)$, by noticing that $1-\varepsilon=a b / V_{\text {total }}$, it is easy to show that the hydraulic diameter can be simplified into a function of the cylinder width 'a', the apex angle and the proposity

$$
D_{h}=\frac{4 \varepsilon V_{\text {total }}}{A_{\text {cont }}}=\left(\frac{\varepsilon}{1-\varepsilon}\right) a \cos (\alpha / 2)
$$

It should be noticed that from a numerical perspective the periodic domain is not the smallest periodic pattern since an additional axial symmetry can be considered along the line passing at the two front and rear apex angles. The choice of the current periodic domain was initially motivated to enable further study of side wall boundary effects in the channel (complete adherence condition on one side). But this was finally out of the scope of the present study.

\section{DEVICE MICRO-FABRICATION}

The fabrication of the device is based on MEMS techniques. First, the pattern of the channel is transferred from a chromium mask onto a $140-400 \mu m$ thick silicon substrate by lithographical steps (spin coating of a S1818 positive photoresist and UV irradiation of $30 \mathrm{~mJ} / \mathrm{cm}^{2}$. ) Then, the pattern of the exchanger with the cylinders matrix is etched using a customized Bosch deep reactive ion etching process (DRIE). As shown in Fig. 3, by controlling the number of cycles, passivation coating, etching and cooling steps durations, a special recipe was established to provide 
structures with a high aspect ratio $(H / a)$ and vertical walls. An overview of the sample matrix, observed with an optical microscope with the same magnification factor of $\times 20$, is presented in Fig. 4. As shown in this figure, for a given cylinder spacing 'e' the increase of the porosity induces a decrease of the column sizes ('a','b').

Finally, the silicon channel is anodically bonded to a Pyrex $1 \mathrm{~mm}$ thick cap that has been preliminary drilled at its extremities for inlet and outlet pipe connexions (see Fig. 1). The sealing between the Pyrex cap and the inlet/outlet holes is insured by using stainless steel slices equipped with vitton O-rings.

The depth of the channel depends on the number of etching cycles. Two kinds of samples have been micro-machined: for the first one the etched depth $H$ is lower than the silicon substrate thickness $(100 \leqslant H \leqslant 190 \mu m$, silicon/Pyrex samples); while for the second one the silicon substrate, previously bonded to a second Pyrex cap, is etched through its entire thickness, making possible the production of transparent Pyrex/Silicon/Pyrex samples.

Dimensions after etching may be different from those of the original mask because of the undesirable side wall etching effects. The real dimensions are determined by analysing a set of optical microscopy photographs. Using the gray level histogram, a threshold is manually adjusted on the gray level images from the optical microscope to binarise the image such that diamondshaped cylinders are put in white while background is in black (empty areas). Then, to accurately characterize the geometry of the etched samples, a conventional images treatment is used for labelling detected objects and extract data statistics [35]. An example of image used for the measurements of the sample dimensions is presented in Fig. 4e. With this method, the 'bounding box' gives the access to 'a' and 'b' values for one cylinder while vertical and horizontal distance between cylinders centroids give the measurements of the periodic pattern sizes $L_{L}=b+2 \Delta x$ and $L_{T}=a+2 \Delta y$. Finally, the arithmetic average of those measurements is considered and the other parameters such as 'c', 'e', ' $\varepsilon$ ' or $D_{h}$ are then calculated using relations introduced in the previous section.

The channel depth has been measured using two methods : in the first one, with a profilometer in an area of the channel without any cylinder (just before or after the porous area); in the second 
one, some samples of the batch are cut crosswise and the depth of the channel is measured by scanning electron microscopy (see Fig. 3, c). The etch depth inside the porous media have been also checked by focusing consecutively on the top and on the bottom of cylinders with an optical microscope equipped with a vernier. Table 1 lists the geometric parameters of the 11 samples micro-fabricated for this work.

\section{FLOW CHARACTERISATION}

The flow and pressure drop have been investigated by varying the Reynolds number $R e$ of the exchanger, calculated as

$$
R e=\frac{\rho U D_{h}}{\mu}
$$

The pressure drop between the inlet and outlet of the flow is then quantified by calculating the Darcy-Weisbach coefficient

$$
f=\frac{D_{h}}{L} \frac{\Delta P}{1 / 2 \rho U^{2}} \quad \Delta P=P_{\text {in }}-P_{\text {out }}
$$

$L$ is the exchanger length ( $L=L_{L}$ for simulations), $U$ is the average flow velocity calculated in the experiment. This formulation based on the linear pressure drop is more adapted to compact heat exchangers than considering singular pressure drop per cylindere row (see for example [22]). Our results have been also analysed using the Poiseuille number defined as the product of the pressure drop coefficient with the Reynolds number $P o=f \times R e$.

During our numerical simulations, the flow around a single cylinder inside the matrix has been also characterized by the corresponding drag coefficient. The drag coefficient is calculated from the drag force $\mathbf{F}_{d}$ by integrating the pressure and viscous stress $\tau$ on the cylinder surface 


$$
C_{D}=\frac{\left\|\mathbf{F}_{d}\right\|}{A_{p}(1 / 2) \rho U^{2}}=\frac{1}{A_{p}(1 / 2) \rho U^{2}}\left\|\int_{S}(-p \cdot \mathbf{n}+\overline{\bar{\tau}} \cdot \mathbf{n}) d S\right\|
$$

with $\mathbf{n}$ the unit vector normal to the cylinder surface, $A_{p}=a H$ corresponds to the projected surface area based on the product of the cylinder width with its height. The drag coefficient variation is analysed with respect to the Reynolds number of the cylinder $R e_{D}$ characterizing the flow at the scale of one cylinder and defined as follow

$$
R e_{D}=\frac{\rho U a}{\mu}
$$

\section{EXPERIMENTAL INVESTIGATIONS}

\section{Experimental setup}

Experiments were performed with helium gas as the working fluid. Fig. 5a shows a diagram of the experimental set-up. Helium gas is supplied by a high pressure gas bottle (200 bar). In order to protect the rest of the experimental assembly, the gas is expanded in a first buffer before passing through the sample. The gas flow and the second buffer pressure are manually controlled by three precision needle valves. The gas flow rate and the inlet-outlet pressures are recorded by a thermal mass flow meter (BrooksDelta, $0.55 \mathrm{NL} / \mathrm{min}$ ) and two pressure transducers (Keller PR33X, 0 to 20 bar). Two k-type thermocouples are also installed inside the capillaries located upstream and downstream the channel, to record the inlet and outlet temperatures. By using the ideal gas law to estimate the density $(\rho=P M / R T)$ and by considering the mass flow conservation along the exchanger $(\rho U S=$ const), it is easy to demonstrate that the ratio between outlet and inlet velocities is simply $U_{\text {out }} / U_{\text {in }} \propto\left(P_{\text {in }} / P_{\text {out }}\right)\left(T_{\text {out }} / T_{\text {in }}\right)$. In present experiments, temperature measurements show that the ratio $T_{\text {out }} / T_{\text {in }}$ remains close to unity $\left(0.99 \leqslant T_{\text {out }} / T_{\text {in }} \leqslant 1.11\right)$. Thus, in order to keep the flow density quite constant and to avoid large velocity variations along the ex- 
changer in our installation the ratio of inlet and outlet pressure has been kept as close as possible to unity. To do that the outlet pressure in the second buffer tank was kept at around 8-10 bar for a pressure drop globally lower than 2 bar (one experiment at 3 bar of maximum). As a result, the velocity of the flow in the exchanger can be calculated directly from the measured mass flow rate at the inlet as follow $U=Q m /\left(\rho S_{\text {totale }} \varepsilon\right)$. The averaged gas density $\rho$ and also average viscosity $\mu$ are calculated from the average inlet/outlet temperature $T_{m}$ and pressure $P_{m}$ using respectively the ideal gas law $\rho=P_{m} M / R T_{m}$ and the correlation proposed by Petersen [36] (Eq. 6.1 in the paper).

As shown in Fig. 5b, the assembly has been designed to facilitate the replacement of samples. The metal structure holds the fluidic connections on the inlet and outlet ports. In the experiments presented here with gaseous helium as the working fluid, it was verified that the Knudsen number remains small enough ( $K \approx 0.015$ ) to avoid slip-like condition at the wall, so the fluid is considered as a continuum [37]. It can also be noted that the regular pressure drop induced in the empty parts of the channel $(\approx 12 \mathrm{~mm})$ has been subtracted from the measured pressure drop [38]. The relative part of this regular pressure drop corresponds to less than $2 \%$ of the total measured pressure drop so that the total measured pressure drop is mainly generated by the cylinders.

\section{Preliminary validation}

In order to check the validity of measurements, some empty channels (without porous region) were micro-machined using $\mathrm{KOH}$ wet anisotropic etching. This method enables to produce channels with a perfectly well defined trapezoidal shape cross section.

Channels were etched with a small depth to obtain a sufficiently high pressure drop together with a gas flow (10 $\leqslant H \leqslant 30 \mu m, W=2 \mathrm{~mm}$ width ). With such a low channel aspect ratio $(H / W<2 \%)$, the flow inside these calibration channels is similar to the one between two parallel plates. The results presented in Fig. 6, show clearly a good agreement with the theoretical friction factor expected for such a reference flow $(f=96 / R e)$. At small Reynolds numbers large error bars are associated to the friction factor. This is due to the larger uncertainty in the measurement of the flow rate at low Reynolds numbers, typically for $R e<10$. For $R e>10$, the relative uncertainty on the measurement of the Reynolds number for experiments with empty channels or channels with 
cylinders is lower than $10 \%$.

\section{Experimental results}

\section{Experimental friction factor}

Experimental measurements of the friction factor were performed for 11 samples on a Reynolds number range of $1<R e<30$. The channels were tested under the same outlet pressure conditions $(\approx 6.7 \mathrm{bar})$ and under the same average gas temperature $\left(\approx 20^{\circ} \mathrm{C}\right)$. The flow rate range is $0.02 \leqslant Q_{v} \leqslant 0.54 \mathrm{NL} / \mathrm{min}$ while the measured pressure drop range is $0.070 \leqslant \Delta P \leqslant 3.0 \mathrm{bar}$. An example of friction factors obtained for samples with the same $e \approx 20 \mu \mathrm{m}$ and different porosities, is reported in Fig. 7 as a function of the Reynolds number. As expected, it is first observed that at low Reynolds number the friction factor evolves as $f=P o / R e$. Each sample with a given porosity can thus be characterized by one Poiseuille number. As previously for friction factor measurements inside empty channels, a high inaccuracy of measurement is obtained for Reynolds number below 10. That is why the Poiseuille number of each sample has been estimated only for data with $R e>10$ insuring an uncertainty on its measurement lower than $20 \%$.

Secondly, it is found that globally the friction factor tends to increase with the porosity $\varepsilon$ for a fixed value of the apex angle (here $\alpha \approx 33^{\circ}$ ). In the literature, the dependency of the friction factor on the porosity for a porous medium in regenerator applications was first mentionned by Gedeon \& Wood [39] but not proved experimentally (Eq. 2.3 in the report of the reference). Even if this behaviour was not clearly noticed by the authors, the increase of the friction factor with $\varepsilon$ was also present in numerical results presented by Costa et al. [40] (Fig. 17 in the paper). In that reference, the authors proposed a single global correlation for four different porosities $(\varepsilon=$ $0.52,0.60,0.64,0.72)$. Nevertheless, it is important to mention that the increase of 'f' with $\varepsilon$ does not mean that increasing the porosity generates higher pressure drop since many parameters can be affected by $\varepsilon$.

The experimental Poiseuille numbers are plotted versus the porosity in Fig. 8 (* symbols) and are reported in the last column of Tab. 1. These experimental data are compared to the results of numerical simulations presented below. For an apex angle $\alpha \approx 33^{\circ}$, both numerical and experimental results display a slight increase of the friction factor when increasing the porosity. 
The small discrepancies between the experimental measurements and the numerical results can be induced by sample border effects (walls) so that the flow is not completly 2D. In all the cases, the Poiseuille number is always higher or equal to the limit given for a flow between two parallel plates $(P o=96)$. This behaviour will be understood in section "Parallel-plates flow analogy" by considering the flow at the scale of the channel formed between cylinders.

Our results are in agreement with the main observation made by Sparrow \& Grannis on the fact that at low apex angle, the Poiseuille number remains nearly constant for $\varepsilon \leqslant 0.60$ (Fig. $11 \& 12$ in [22]). However, a direct comparison with this previous work is made difficult, because the authors do not use exactly the same velocity scale as ours for the calculation of the Reynolds numbers and the friction factors. Nevertheless, the present work displayed the same order of magnitude of Poiseuille numbers than what has been presented by this team ( $P o \approx 100$ for $\alpha=45^{\circ}$ and $\varepsilon \leqslant 0.70)$.

Moreover, one of the most important result of this work is that the diamond-shaped array geometry is found to generate experimentally lower Poiseuille numbers than conventional solutions used to design regenerators. As reported in Fig. 8, measured Poiseuille numbers are found to be smaller that stacked woven wire matrices ( $P_{0}=123$, Costa et al. [41] for $\left.1 \leqslant R e \leqslant 400\right)$ or stacked sphere matrices randomly arranged ( $P_{O}=133$, Ergun [42] for moderate Reynolds number, see [43] appendix B) for $\varepsilon \leqslant 60-70 \%$. This result confirms that well controlled geometries can improve regenerators pressure losses and more globally heat exchanger performance.

Lastly, another important new result emerging from Fig. 8 is that contrary to the experimental results presented by Vanapalli et al. [20] for sinus $\left(P_{O} \approx 29.65\right)$ or ellipse-shaped $(P o \approx 26.98)$ cylinders, our experimental results do not display Poiseuille numbers below the parallel-plates limit $(P o \leqslant 96$ in our case). There is no reason that the Poiseuille number should be lower than the squared cross section channel limit $\left(P_{O}=56.9\right)$. The result of Vanapalli et al. maybe a consequence of the singular pressure corrections applied to the porous media extremities, or of an inaccurate hydraulic diameter used that does not take into account the additional friction on the bottom and top walls $\left(A_{\text {cont }}^{\prime}\right)$.

Those interesting observations make very promising the use of this geometry for the design 
of exchangers with low pressure drop, and complementary studies on heat transfer efficiency with such a geometry will have to be performed. The dimensional analysis presented now will help to understand that the Poiseuille number depends on porosity before being confirmed by the numerical simulations.

\section{Dimensional analysis}

To confirm experimental observations and before developping numerical simulation, a dimensional analysis was applied in order to identify the main dimensionless numbers that influence the friction factor in the studied geometries. In our configuration the pressure drop $\Delta P$ through the sample can be influenced by the seven following parameters: $U, \rho, \mu, a, b, D_{h}$ and $L$ (the porous area length). The dimensions of those parameters are recalled in Tab. 2. Note that the porosity $\varepsilon$ or the interchannel width $e$ are not included since they are both linked to parameters a, b, $D_{h}$. In addition, the channel height $H$ is not taken into account since we consider here a 2D case with $a / H<<1$ and $b / H<<1$. As shown in Tab 2, there is $n=8$ parameters and $m=3$ fundamental dimensions (L, T, M). As a result, applying the Vaschy-Buckingham theorem [44, 45], our system is controlled by $n-m=5$ dimensionless numbers that can first be expressed as

$$
\begin{aligned}
& \Pi_{1}=\Delta P U^{-2} \rho^{-1} D_{h}^{0}=\frac{\Delta P}{\rho U^{2}} \\
& \Pi_{2}=\mu U^{-1} \rho^{-1} D_{h}^{-1}=\frac{\mu}{U \rho D_{h}} \\
& \Pi_{3}=a U^{0} \rho^{0} D_{h}^{-1}=\frac{a}{D_{h}} \\
& \Pi_{4}=b U^{0} \rho^{0} D_{h}^{-1}=\frac{b}{D_{h}} \\
& \Pi_{5}=L U^{0} \rho^{0} D_{h}^{-1}=\frac{L}{D_{h}}
\end{aligned}
$$

Among those numbers, it is important to note that in our case, the condition $\Pi_{5}=L / D_{h} \rightarrow \infty$ is always verified. Our system can thus be reduced to 4 dimensionless numbers that can be rearranged as 


$$
\begin{aligned}
\frac{\Pi_{1}}{\Pi_{5}} & =\frac{\Delta P D_{h}}{\rho U^{2} L}=f \\
\Pi_{2}^{-1} & =\frac{U \rho D_{h}}{\mu}=R e \\
\Pi_{3} & =\frac{a}{D_{h}}=(1-\varepsilon) /[\cos (\alpha / 2) \varepsilon /] \\
\Pi_{3} / \Pi_{4} & =\frac{a}{b}=\tan (\alpha / 2)
\end{aligned}
$$

Consequently, the dimensional analysis confirms that the friction factor depends on the Reynolds number Re, on the apex angle $\alpha$ (or $\tan (\alpha / 2))$ and on the porosity. For a fixed value of the apex angle, the dimensional analysis confirms that the friction factor is influenced not only by the exchanger Reynolds number but also by the porosity value. One can note that this parameter can be also interpreted as the effect of the variation of the cylinder Reynolds number since $\Pi_{3} / \Pi_{2}=U \rho a / \mu=R e_{D}$. It is important to notice that in the literature, it is known that the pressure drop in staggered arrangements depends on the center-to-center longitudinal and transversal distances $L_{L}$ and $L_{T} / 2$ (see for example [31]). Since in our geometry the constraint $\Delta y=e$ makes a direct link between longitudinal and transversal distances, the porosity quantifies here the distances between column center.

Our experimental measurements show that the Poiseuille number increases with the porosity. To go further in the possible use of diamond-shaped arrays for micro regenerators geometry, it is now important to confirm and understand this behaviour and to investigate the effect of varying the apex angle. This is the objective of the next part of the article where those open questions are addressed using numerical simulations.

\section{NUMERICAL SIMULATIONS}

In this section, the numerical procedure, the validation of the procedure and results are presented for a 2D flow through a diamond-shaped cylinders array. 


\section{Governing equations}

One considers the numerical simulation of a flow through a cylinder matrix with a volume averaged mean velocity ' $U=(1 / V) \iiint u_{x} d v$ ' along the main flow axis. The cylinders aspect ratio is considered to be small enough so that the flow is in 2 dimensions (i.e. $a / H<<1$ ). Due to the Reynolds number range, the flow regime is laminar. The local velocity 'u' and pressure fields ' $p$ ' are given by solving numerically the Navier-Stokes equations for an incompressible Newtonian fluid

$$
\begin{aligned}
\nabla \cdot \mathbf{u} & =0 \\
\rho \mathbf{u} \cdot \nabla \mathbf{u} & =-\nabla p+\nabla \cdot \overline{\bar{\tau}}+S_{0}
\end{aligned}
$$

where $\overline{\bar{\tau}}=\mu\left(\nabla \mathbf{u}+\nabla^{T} \mathbf{u}\right)$ is the viscous contribution of the stress tensor, $\rho$ and $\mu$ are respectively the fluid density and the dynamic viscosity. The flow is generated by imposing a source term $S_{0}=\partial p / \partial x$ as a negative pressure variation along the $\mathrm{x}$-axis matrix and using periodic boundary conditions for both velocity and pressure as detailed in the next section.

\section{Numerical procedure}

The conservation equations are solved with the finite volume method in 2D with OpenFoam using a double-precision writing format [46]. For solving the Navier-Stokes equations, an upwind second order scheme and a centered second order scheme are used for the spatial discretization of respectively the advective and the diffusive fluxes. The pressure and velocity fields are linked by the SIMPLE algorithm. The convergence of the flow calculation is achieved when the drag coefficient is stable and when the residuals are lower than $10^{-7}$.

The calculation domain is presented in Fig. 9a. It corresponds to a periodic pattern with periodic boundary conditions at the inlet and at the outlet for the velocity and the pressure (i.e. $\mathbf{u}(x=0, y)=\mathbf{u}\left(x=L_{L}, y\right)$ and $\left.p(x=0, y)=p\left(x=L_{L}, y\right)\right)$. An adherence condition is imposed at the cylinder walls $(\mathbf{u}=0)$. A symmetry boundary condition is set on the upper and bottom sides. 
Some preliminary simulation tests on a domain corresponding to a concatenation of five periodic patterns have shown that the periodic boundary conditions on velocity and on residual pressure are reached as soon as the second periodic box is passed for present Reynolds range.

An example of the grid refinement strategy is shown in Fig 9b for a coarse grid. Around the cylinder a refined body fitted rectangular mesh is used. The grid-independence of results has been checked for each case. The size of the grids used in this work is detailed in Tab. 3. Globally, the number of cells between columns faces varies as $50<N e<300(N e=N \Delta y)$. The number of cells along the cylinder surface (2c) is in the range $62<2 N c<180$. And the number of cells between two consecutive apex angles is fixed to be $2 N \Delta x=10$. The number of cells indicated here are linked to segments presented in Fig. $2(c, e, \Delta x, \Delta y)$. In the present simulations, since the Reynolds number remains low the boundary layer are thick. Thus the smallest size of the cell at the cylinder surface is not fixed from a boundary layer constraint but by respecting a cell aspect ratio between 2 and 4 and checking the result independence to the grid refinement.

\section{Numerical results and discussion}

The preliminary validation of the numerical procedure was carried out using two test cases. The first test case is the flow between two parallel plates considering a periodic slice. Numerical results show a perfect agreement with the theory with $P o=96$ for $1 \leqslant R e \leqslant 100$. The second validation test case is a flow through a staggered arrangement diamond-shaped cylinders matrix with a porosity of $\varepsilon=65 \%$ and an apex angle of $\alpha=45^{\circ}$ as studied experimentally by Sparrow \& Grannis [16]. A correct agreement is globally found with respectively a difference of $3.8 \% .4 .5 \%$ and $15 \%$ for the three measurments presented by the authors (see Fig. 3.5, Tab. 3.2 and Fig. $3.4 \mathrm{~b}$ in [43]).

In this section, the pressure drop is presented for a flow through diamond-shaped cylinders matrix, with variable apex angles $\left(33 \leqslant \alpha \leqslant 90^{\circ}\right)$, porosity $(40 \leqslant \varepsilon \leqslant 90 \%)$ and Reynolds number range $(1 \leqslant R e \leqslant 30)$ matching the experimental study. Then, to understand Poiseuille variations, our results are also analysed at the scale of a channel flanked by neighbouring cylinders and at the scale of one cylinder in the matrix. 
Pressure drop at the exchanger scale

Some examples of velocity fields obtained by the simulations for $R e=1-30, \alpha=33-60-90^{\circ}$ are presented in figures 10 and 11 for $\varepsilon=40 \%$ and $\varepsilon=80 \%$ respectively. In Fig. 10 for $\varepsilon=40 \%$, at $R e=1$ (LHS) it can be observed that whatever the apex angle is, the flow displays a velocity profile very close to the parabolic velocity profile that is usually found for a parallel-plates flow. As shown in the same figure, at $R e=30(\mathrm{RHS})$, the velocity profiles became a bit asymmetric when increasing the apex angle. A velocity deficit is observed just after the top and bottom equator angles but no recirculation is formed at the rear of the cylinder. Present velocity fields are in agreement with previous observations of [22] or [26, 27] $\left(\alpha=43-60^{\circ}\right.$ and $\left.\varepsilon=40-60-80 \%\right)$. In Fig. 11 for $\varepsilon=80 \%$, at $R e=30(\mathrm{RHS})$ it is found that the increase of the apex angle generates stronger flow asymmetry compared to $\varepsilon=40 \%$. As a conclusion, the analysis of velocity fields demonstrates that at low Reynolds number, a low porosity and a low apex angle support the establishment of a velocity profile close to the parabolic one expected for creeping flow between two parallel plates.

The corresponding evolutions of friction factor as a function of the Reynolds number for $\alpha=33$ and $90^{\circ}$ are shown in Fig. 12. In agreement with the experiments, it is verified that the calculated friction factor increases with the porosity $\varepsilon$ and remains always higher than the one expected for the flow through a flat rectangular channel $\left(f \geqslant 96 /\right.$ Re or $\left.P_{o} \geqslant 96\right)$. In addition, numerical simulations indicate also that the friction factor can increase substantially with the apex angle. This may be explained by the flow deviation induced by an $\alpha$ enlargement. An increase of the pressure drop due to flow deviation has been also reported experimentally by [47] for the case of a flow following a sinusoidal trajectory. However, the dependency of the friction factor on the porosity is for the moment not clearly explained.

Globally at low Reynolds number the Stokes flow is clearly verified $(f \propto 1 / R e)$ so that a Poiseille number $P o=f R e$ can be affected to each geometrie. Poiseille numbers obtained from the numerical simulations are reported in Table 4 and plotted in Fig. 8. Considering the experimental errorbars with an uncertainty around $20 \%$ on the experimental Poiseuille number, a good agreement is found between the numerical results and the experimental measurements for $\alpha=33^{\circ}$. To 
justify and to better understand the effect of the porosity and apex angle on the Poiseuille number, we propose now to analyse the flow at different scales.

\section{Parallel-plates flow analogy}

It is a common practice, for porous media, to make the analogy with channelling flows to try to understand the effect of the different parameters [42, 48]. This approach is particularly justified in our geometry considering previous observation on the velocity field. The channel formed between cylinders, corresponding to the area limited by red dashed lines in Fig. 2, is now considered for applying the parallel-plates flow analogy. Thus, in order to make possible the analysis at the scale of the channel formed between cylinders, the length $L_{L}^{\prime}$, which corresponds to the averaged flow path length, is now introduced (see Fig. 2).

By considering the mid distance between the top and bottom walls, an estimation of the flow path length can be given as following (for more details see [43])

$$
L_{L}^{\prime}=4 \Delta x \sqrt{1+(a /(2 b))^{2}}+2(b / 2-\Delta x) \sqrt{1+(a / b)^{2}}
$$

As for the whole exchanger, the hydraulic diameter, the Reynolds number and the friction factor corresponding to this channel can be defined as

$$
D_{h}^{\prime}=4 e H /(2(e+H))=4 e /(2(e / H+1)) \approx 2 e
$$

$$
R e^{\prime}=U D_{h}^{\prime} / \nu \approx \operatorname{Re} D_{h}^{\prime} / D_{h}=\operatorname{Re} 2 e / D_{h}
$$




$$
f^{\prime}=\frac{D_{h}^{\prime}}{L_{L}^{\prime}} \frac{\Delta P}{1 / 2 \rho U^{2}}=\frac{D_{h}^{\prime}}{L_{L}^{\prime}} \frac{L_{L}}{D_{h}} f=\frac{D_{h}^{\prime}}{D_{h}} \frac{L_{L}}{L_{L}^{\prime}} f
$$

The channel friction factor ( $\left.f^{\prime}\right)$ can thus be calculated directly from previously defined friction factor for the whole exchanger (f) and from the length ratios $L_{L}^{\prime} / L_{L}$ and $D_{h}^{\prime} / D_{h}$. Those two length ratios are plotted in Fig. 13 as a function of the porosity for different apex angles.

A first attempt to apply the 'channel' flow analogy for diamond-shaped cylinders array was proposed by Grannis \& Sparrow [22]. They did not take into account real flow path $\left(L_{L}^{\prime}\right)$ and only use the porous media hydraulic definitions. In porous media the length ratio $L_{L}^{\prime} / L_{L}$ corresponds to the so-called tortuosity that compares the mean real path length of the flow through the pores network to the length of the porous matrix. By definition this ratio is larger or equal to unity. In Fig. $13 a$, one can show that for present geometry even if the range of apex angle is large $\left(33 \leqslant \alpha \leqslant 90^{\circ}\right)$ the tortuosity remains lower than 1.4. Moreover the tortuosity seems to be significantly affected by the porosity only for $\alpha>50^{\circ}$. In addition, decreasing the apex angle makes this ratio very close to unity. As a result one particularity of the diamond-shaped cylinder matrix regenerators is to be characterized by a low tortuosity. In the same time, as depicted in Fig. 13b, the hydraulic diameter ratio $D_{h}^{\prime} / D_{h}$ is found to decrease when the porosity increases, and to be lower than unity. For a given porosity and total volume, this ratio is proportional to the corresponding wetted surfaces ratio so that $D_{h}^{\prime} / D_{h} \propto A_{\text {cont }} / A_{\text {cont }}^{\prime}$. Since in our geometry, the fluid is not wetting all the border of the channel formed between cylinders (area delimited by a red dashes line in Fig. 2) the wetted surface $A_{\text {cont }}$ is smaller than the one expected in a straight channel having the same length $L_{L}^{\prime}$ so that $A_{\text {cont }}<A_{\text {cont }}^{\prime}$ and $D_{h}^{\prime} / D_{h}<1$. The observation that in both numerical and experimental approaches the Poiseuille number tends to the parallel-plates flow limit $P o=96$ when decreasing the porosity and apex angle, is therefore explained by the behaviour $D_{h}^{\prime} / D_{h}(\alpha \rightarrow 0, \varepsilon \rightarrow 0) \rightarrow 1$ and $L_{L}^{\prime} / L_{L}(\alpha \rightarrow 0, \varepsilon \rightarrow 0) \rightarrow 1$. In those conditions the flow is similar to the one in an assembly of parallel flat channels with length $L_{L}=L_{L}^{\prime}$ and hydraulic diameter $D_{h}=D_{h}^{\prime}=2 e$. In addition, when $\varepsilon \rightarrow 1$, this behaviour should also be found. For such conditions, in Eq. 2, the wetted surface in 
contact with the top an bottom channel walls $A_{\text {cont }}^{W}$ is predominant compared to the wetted surface in contact with the column $A_{\text {cont }}$ and the hydraulic diameter tends to $D_{h}=2 H$. If the aspect ratio is low $H / W<<1$, the Poiseuille number when $\varepsilon \rightarrow 1$ should also be $P o=96$ and the main channel flow become similar to the parallel-plates flow.

It is now possible to estimate the Poiseuille number $\mathrm{Po}^{\prime}$ corresponding to the pressure loss at the scale of the channel formed between cylinders as follow

$$
P o^{\prime}=R e^{\prime} f^{\prime}=\left(\frac{D_{h}^{\prime}}{D_{h}}\right)^{2} \frac{L_{L}}{L_{L}^{\prime}} f R e=\left(\frac{D_{h}^{\prime}}{D_{h}}\right)^{2} \frac{L_{L}}{L_{L}^{\prime}} P o
$$

The evolution of $\mathrm{Po}^{\prime}$ as a function of the porosity is presented in Fig. 14a. It is first observed that the Poiseuille variation is much lower than previously in Fig. $8\left(95<P o^{\prime}<140\right.$ whereas $107<P o<357)$ and that for a given apex angle $\alpha$ the value of $P o^{\prime}$ is weakly affected by the variation of the porosity. To verify this assertion, the average value of $P o^{\prime}$ for a given angle $\alpha$ $\left(<P o^{\prime}>\right)$, the standard deviation compared to this value $\left(S T D\left(P o^{\prime}\right)\right)$ and the maximal absolute difference with this average value $\left(\Delta \operatorname{Max}\left(\left|P o^{\prime}-<P o^{\prime}>\right|\right)\right)$ have been reported in Tab 5 . For the simulations presented here, the standard deviation and the maximal difference are both lower than $6 \%$. As a consequence, the quasi stagnation of the Poiseuille number of the channel $P o^{\prime}$ versus $\varepsilon$ show us that the dependence of the Poiseuille number Po on the porosity of the exchanger is mainly explained by the change of the scale considered to analyse the results (exchanger scale / channel scale).

In addition, as shown in Fig. 14b, it is therefore possible to plot the evolution of the average Poiseuille number $\left\langle P o^{\prime}>\right.$ as a function of the apex angle $\alpha$. The evolution of $\left\langle P o^{\prime}>\right.$ can be fitted by

$$
<P o^{\prime}>=41[\tan (\alpha / 2)]^{2}+96
$$


with $\alpha$ in radian. This relation was constructed considering that, as mention previously, < $P o^{\prime}>(\alpha \rightarrow 0)=96$ and using the aspect ratio $\tan (\alpha / 2)=a / b$ that reflects the velocity field deviation intensity induced by the apex angle value. The increase of $\left\langle P o^{\prime}\right\rangle$ with the apex angle can be explained by the fact that the highest the apex angle is, the strongest the flow deviation and associated pressure loss are. In Fig. 14b, it can be noticed that the fact of not having data for $\varepsilon$ larger than $70 \%$ for $\alpha=40^{\circ}$ and $50^{\circ}$ may explained that corresponding values for $<P o>$ are a bit higher than the curve given by Eq. 24.

This data analysis have the advantage to enable the estimation of $\operatorname{Po}(\alpha, \varepsilon)$ values from an average value of $P o$ that is only a function of $\alpha$ (Eq. 24). From Eq. 23 and Eq. 24, it becomes now possible to estimate the effect of the apex angle and to use the hydraulic diameter ratio and the tortuosity to estimate the effect of the porosity on the Poiseuille number considered at the scale of the whole exchanger. The results reported in Fig. 8 (dash lines) are found to be in good agreement with the numerical simulations, especially at low apex angle.

\section{Collective effect on drag coefficient}

In the literature on collective effect on drag, it is claimed that the variation of the drag exerted on one cylinder in an array can be influenced by two contributions. If two cylinders are aligned in the direction of the flow, the decrease of the distance between them tends to reduce the drag : this is the longitudinal contribution (see for example [49] ). If two elements are now aligned perpendicularly to the flow direction, then it is admitted that the drag of both cylinders increases if they get closer because of the fluid blockage and the induced increase of friction: this is the transversal contribution (see for example [50] or [51]). Consequently, the drag exerted on one cylinder results from the interaction of these two contributions.

Some example of the typical evolution of the drag coefficients of a cylinder in the matrix are plotted in Fig. 15 for $\alpha=33$ and $90^{\circ}$. Globally, for a given angle $\alpha$, it is observed that the drag increases systematically when the porosity decreases and the spacing between cylinders is reduced. In addition, the correlation found by Sochinskii et al. [52] for a single diamond-shaped cylinder is also reported in this figure. As for an array of particles [53, 42], it is found that the drag for one cylinder in a matrix is always larger than the one expected for a single cylinder. This 
behaviour is also in agreement with the literature on collective effect on drag force for a staggered array of cylinders [2]. We can note that, at low Reynolds numbers, the drag coefficient seems to remains inversely proportional to the cylinder Reynolds number even if the porosity is reduced.

To understand the link between the collective effect on drag and the friction factor corresponding to the pressure loss in the cylinder matrix, it is possible to write the momentum global balance for a volume corresponding to the present periodic pattern. The calculation developed in APPENDIX A shows that the pressure loss is induced by the force applied on the cylinders so that the drag and friction coefficients are linked by the relation

$$
C_{D} \approx f \frac{L_{L} L_{T}}{D_{h} 2 a}=\left(\frac{P o L_{L} L_{T}}{2 D_{h}^{2}}\right) \frac{1}{R e_{D}}=\left(\frac{<P o^{\prime}>L_{L}^{\prime} L_{T}}{2 D_{h}^{\prime 2}}\right) \frac{1}{R e_{D}}
$$

This result, usually presented as Eq. 30, is also found by Larson \& Higdon [54] or Van der Hoef et al. [55] and has been verified in our simulations with a maximum difference of $3 \%$.

From the begining of Eq. 25, it can be shown that the concomitant increase of $f$ and decrease of $C_{D}$ with the porosity can be explained by the strong reduction of the ratio $\left(L_{L} L_{T}\right) /\left(D_{h} 2 a\right) \propto \varepsilon^{-1}$ that is inversely portional to the porosity. Indeed, by considering Eq. 3, $a b /\left(L_{L} L_{T}\right)=1-\varepsilon$ and $\tan (\alpha / 2)=a / b$, this ratio can be simply expressed as

$$
\frac{L_{L} L_{T}}{D_{h} 2 a}=[2 \sin (\alpha / 2) \varepsilon]^{-1}
$$

From Eq. 25 and 24, it becomes now possible to estimate the drag coefficient at low Reynolds numbers. The result is reported as dotted lines in Fig. 15 and is in a very good agreement with our simulation results. Consequently, the analysis of the flow at the scale of the channel formed between cylinders has allowed us to develop a description of an average Poiseuille number, that can be used for the prediction of pressure losses at the scale of the heat exchanger but also for 
the estimation of collective effects on the drag coefficient at the scale of one cylinder.

\section{Comparison to circular cylinders and spherical particles arrays}

This section compares our method and results on Poiseuille numbers with those expected for a square and staggered arrangement of circular cylinders matrix and for conventional spherical particles arrays.

The collective effect on drag for circular cylinders arrays is well documented, and the Poiseuille number of such an arrangement can be calculated directly from the drag force. The detail of the calculations that make the link between the Poiseuille numbers and the drag for a circular cylinders array is presented in the first part of APPENDIX B. The numerical results of Sangani \& Acrivos [2] and the hybrid model of Yeom et al. [6] constructed from experimental measurements are reported in Fig. 16. Our first observation is that the Poiseuille number obtained using a circular cylinders array is always higher than the one using a diamond-shaped cylinders array, except for $\alpha=90^{\circ}$. This result allows to appreciate the potential of using a diamond-shaped cylinder geometry for a low pressure loss design for heat exchangers operated at low and moderate Reynolds numbers. In contrast with our results, the obtained Poiseuille variation with the porosity for circular cylinders exhibits a 'U' shape reaching a minimum value around $\varepsilon=0.70-75$. The increase of the Poiseuille number when decreasing the porosity is thought to be the consequence of the strong variations of the cross section of the flow along its path, generating acceleration/deceleration when decreasing the porosity. Furthermore the increase of the Poiseuille number when increasing the porosity is found to be in agreement with our results for $\alpha=90^{\circ}$ and typically for $\varepsilon>0.8$. This behaviour is first explained by the fact that when the porosity gets close to unity the flow around the cylinders gets close to the flow around a single cylinder whatever the arrangement is. Secondly, as reported recently by Sochinskii et al. [52] the drag for a circular cylinder is very close to the one obtained from a diamond-shaped cylinder with an apex angle of $\alpha=90^{\circ}$. As a result, the arrangement of circular or $90^{\circ}$ diamond-shaped cylinders gives similar drag law and Poiseuille number when the porosity tends to unity. This is confirmed by the fact that the dimensionless drag force predicted by the relation proposed by Yeom et al. [6] for $\epsilon=0.99$ with a square array of circular cylinders ( $F / \mu U=3.16)$ is close to the dimensionless drag force experienced by a single diamond-shaped 
cylinder with $\alpha=90^{\circ}$ at low Reynolds number $\left(F / \mu U=\left[\left(C_{D} / 2\right)\left(D_{h}\right) \rho U^{2}\right] /(\mu U H)=6.98 / 2=3.49\right.$, [52]).

In addition, as mentioned previously, the Ergun correlation is dedicated to randomly arranged spherical particles arrays at moderate Reynolds numbers. In the literature, the drag force on well controlled arrangements of spherical particles is also well documented. The details of the calculations that make the link between the Poiseuille numbers and the drag for spherical particles arrays are presented in the second part of APPENDIX B. The numerical results of Sangani \& Acrivos [56], valid for low Reynolds number, are now reported in Fig. 16 for simple, body centred and face centred cubic arrays of spherical particles. It is found again that, typically for $\varepsilon \leqslant 70 \%$, those specific sphere networks tend to generate higher Po than diamond-shaped cylinders arrays but lower Po than circular cylinders arrays. The Poiseuille number curves for body and face centred cubic arrays still exhibit a 'U' shape. Meanwhile for the simple cubic array, Po shows a monotonous increase with the porosity. As for the circular cylinders arrays, at high porosity $(\varepsilon>80 \%)$ the type of arrangements do not influence the Poiseuille number that increases strongly with the porosity. In fact, for a very dilute particle flow at low Reynolds numbers, the drag coefficient of the particles is close to the one of a single particle $\left(C_{D}=24 / \operatorname{Re}_{D}, F=3 \pi U \mu D\right.$ [57]). Rearranging equations from APPENDIX B, with such a drag law, one finds easily that at high porosity Poiseuille numbers follow

$$
\operatorname{Po}(\epsilon \rightarrow 1)=\frac{K \varepsilon^{2}}{1-\varepsilon}
$$

with $K=16$ for spherical particles arrays. As reported in Fig. 16, this asymptotic behaviour is in agreement with the results of Sangani \& Acrivos [56]. The identification of the behaviour of $P o$ for a dilute flow with the circular cylinders arrays is made difficult because of the complexity of available drag laws at low Reynolds numbers. Similarly, for the present geometry with diamond-shaped cylinders arrays, under very dilute conditions the drag experienced by cylinders remains close to the one on a single cylinder whatever the arrangement is $\left(C_{D} \approx 7 / \operatorname{Re}_{D}\right.$ for 
$R e_{D} \leqslant 10$ [52]). The rearrangement of the equations show that the asymptotic behaviour of very dilute diamond-shaped cylinders arrays follows the same previous equation (Eq. 27) with $K=14 \tan (\alpha / 2) /\left([\tan (\alpha / 2)]^{2}+1\right)$. As shown in Fig. 16, it is difficult with our results (where the maximum porosity is $90 \%$ ) to verify this limit. Nevertheless, the fact that the $K$ factor increases with the apex angle support the idea that, as observed previously in Fig. 8, Po is less influenced by the porosity when decreasing the apex angle. It is important to note that such a divergence of the Poiseuille number when $\epsilon \rightarrow 1$ could be only observed experimentally for condition close to a unbounded 2D flow $\left(a / H<<1, a / W<<1\right.$ and $\left.A_{\text {cont }}^{W}<<A_{\text {cont }}\right)$. As explained previously, by increasing the porosity if $A_{c o n t}^{W}>>A_{\text {cont }}$ the pressure drop will be controled by the main channel size $(H, W, L)$. 


\section{SUMMARY AND CONCLUSIONS}

In this work, three complementary approaches(experiments, dimensional analysis and numerical simulations) were developped to investigate pressure losses in diamond-shaped cylinders arrays at moderate Reynolds numbers. Experiments have been performed to study the influence of the porosity on the friction factor on a micro-fabricated diamond-shaped cylinders array with an apex angle of $\alpha=33^{\circ}$.

- The measurements exhibited a weak increase of the Poiseuille number with the porosity, confirming a trend that can be found in recent numerical studies of Costa et al. [40].

- The fact that the Poiseuille number depends on the porosity is confirmed by a dimensional analysis.

- Experimental Poiseuille numbers are higher than the value expected from a two parallel plates geometry $\left(P_{o} \geqslant 96\right)$. But they remains lower than those reached by conventional solutions used up to now to design regenerators (spheres and wires stacking arrays or even circular cylinders arrays). That result enhances all the capability of the diamond-shaped geometry for the design of heat exchangers with low pressure drop, enabling the reduction of exchangers hydraulic power.

The flow in diamond-shaped cylinders arrays has been investigated using numerical simulations for a large range of apex angles $33^{\circ} \leqslant \alpha \leqslant 90^{\circ}$.

- The numerical results confirm the increase of the Poiseuille number with the porosity, but also with the apex angle.

- The analysis of the results at the scale of a channel located between two adjacent cylinders, shows that the role of the porosity is mainly explained by the variation of the real flow path length. The contribution of the apex angle variation is the consequence of the pressure drop induced by the flow deviation.

- The analysis of the collective effect on drag is found to be in agreement with the literature and shows an increase of the drag when the distance between cylinder is reduced.

- From a multi-scale analysis, a simple correlation has been identified that enables the description of both the Poiseuille number and the drag coefficient variation for a large range of apex 
angles $33^{\circ} \leqslant \alpha \leqslant 90^{\circ}$ and porosities $40 \leqslant \varepsilon \leqslant 90 \%$ at moderate Reynolds number.

Forthcoming works will be devoted to experimentally characterize the role of the apex angle of the diamond-shaped cylinders on the Poiseuille number for low Reynolds numbers, and to verify that the results match those obtained numerically for $\alpha>33^{\circ}$. The main challenge will be then to investigate numerically and experimentally the heat exchange efficiency for such a geometry.

\section{ACKNOWLEDGMENTS}

The authors would like to thank LANEF laboratory of excellence as well as SBT/CEA and LEGI for supporting this work. We gratefully acknowledge the assistance of Cyrille Bonamy from LEGI for his help in numerical simulations with OpenFoam, of David Garcia and of Julien Inigo from SBT for assembling the experimental installation. We also thank Kabir Bashir Shariff for his helpful involvement during his master internship. Authors would like to also acknowledge the technical staffs of PTA (CEA) and Nanofab (Néel Institute) micro/nanofabrication platforms for the assistance in micro-fabrication of samples. 


\section{NOMENCLATURE}

\section{Roman symbols}

a diamond-shaped cylinder width, $m$

$A_{\text {cont }} \quad$ cylinder wetted surface in contact with the fluid, $m^{2}$

$A_{\text {cont }}^{W} \quad$ channel caps wetted surface in contact with the fluid, $m^{2}$

$A_{\text {cont }}^{\prime} \quad$ wetted surface for the channel considered between cylinders, $m^{2}$

$b \quad$ diamond-shaped cylinder length, $m$

$c \quad$ diamond-shaped cylinder half side length, $m$

$C_{D} \quad$ drag coefficient

D circular cylinder or spherical particle diameter, $m$

$D_{h} \quad$ hydraulic diameter of the exchanger, $m$

$D_{h}^{\prime} \quad$ hydraulic diameter of the channel formed by cylinders, $m$

$e \quad$ distance between cylinders walls and width of channel formed by cylinders, $m$

$f \quad$ friction factor at the exchanger scale

$f^{\prime} \quad$ friction factor considering the channel formed by cylinders

$\mathbf{F}_{d} \quad$ drag force vector for one cylinder, $N$

$F \quad$ drag force magnitude per cylinder length $\left(F=\left\|\mathbf{F}_{d}\right\| / H\right), N / m$

$H \quad$ cylinder height (or etching height), $m$

$L_{T} \quad$ width of the periodic domain, $m$

$L_{L} \quad$ width of the periodic domain, $m$

$L_{L}^{\prime} \quad$ estimation of the real flow path length along the channel in the periodic domain, $m$

$L \quad$ experimental exchanger length section with the diamond-shaped cylinders array, $m$

$p \quad$ local pressure, $\mathrm{Pa}$

Po Poiseuille number at the scale of the exchanger $(P o=f R e)$

$P o^{\prime} \quad$ Poiseuille number at the channel $\left(P o^{\prime}=f^{\prime} R e^{\prime}\right)$

$Q_{m} \quad$ mass flow rate, $\mathrm{kg} \mathrm{s}^{-1}$

$R \quad$ Ideal gas constant $\left(R=8.314 \mathrm{~J} \mathrm{~mol}^{-1} \mathrm{~K}^{-1}\right)$

Re Reynolds number based on the exchanger hydraulic diameter and mean flow velocity $\left(R e=U D_{h} / \nu\right)$ 
$R e^{\prime} \quad$ Reynolds number based on the channel hydraulic diameter and mean flow velocity $\left(R e^{\prime}=U D_{h}^{\prime} / \nu\right)$

$R e_{D} \quad$ cylinder Reynolds number based on the cylinder width and mean flow velocity $\left(\operatorname{Re}_{D}=U a / \nu\right)$

$S \quad$ cross section area, $m^{2}$

$T_{m} \quad$ average inlet/outlet temperature, $K$

u local velocity vector, $m s^{-1}$

$U \quad$ mean flow velocity, $m s^{-1}$

Greek symbols

$\alpha \quad$ apex angle, ${ }^{\circ}$

$\varepsilon \quad$ exchanger porosity

$\mu \quad$ dynamic viscosity, $P$ a s

$\nu \quad$ kinematic viscosity $(\nu=\mu / \rho), m^{2} s^{-1}$

$\rho \quad$ density, $\mathrm{kg} \mathrm{m}^{-3}$

Mathematical symbol

$\|-\|$ vector magnitude

\section{REFERENCES}

[1] Zukauskas, A., 1972. "Heat transfer from tubes in cross flow". Adv. Heat Transfer, 8, pp. 93160.

[2] Sangani, A., and Acrivos, A., 1982. "Slow flow past periodic arrays of cylinders with application to heat transfer". Int. J. Multiph. Flow, 8, pp. 193-206.

[3] Metzger, D., Berry, R., and Bronson, J., 1982. "Developing heat transfer in rectangular ducts with staggered arrays of short pin fins". J. Heat Transfer, 104, pp. 700-706.

[4] Stanescu, G., Fowler, A., and Bejan, A., 1996. "The optimal spacing of cylinders in freestream cross-flow forced convection". Int. J. Heat Mass Transf., 39, pp. 311-317.

[5] Peles, Y., Kosar, A., Mishra, C., Kuo, C., and Schneider, B., 2005. "Forced convective heat 
transfer across a pin fin micro heat sink". Int. J. Heat Mass Transf., 48, pp. 3615-3627.

[6] Yeom, J., Agonafer, D., Han, J., and Shannon, M., 2009. "Low reynolds number flow across an array of cylindrical microposts in a microchannel and figure-of-merit analysis of micropostfilled microreactors". J. Micromech. Microeng., 19, p. 065025.

[7] Li, Q., Chen, Z., Flechtner, U., and Warnecke, H., 1998. "Heat transfer and pressure drop characteristics in rectangular channels with elliptic pin fins". Int. J. Heat Fluid Flow, 19, pp. 245-250.

[8] Uzol, O., and C, C., 2005. "Heat transfer, pressure loss and flow field measurements downstream of staggered two-row circular and elliptical pin fin arrays". J. Heat Transfer, 127, pp. 458-471.

[9] Bahaidarah, H., Anand, N., and Chen, H., 2005. "A numerical study of fluid flow and heat transfer over a bank of flat tubes". Numer. Heat Tr. A-Appl., 48(4), pp. 359-385.

[10] Molki, M., Faghri, M., and Ozbay, O., 1994. "A new correlation for pressure drop in arrays of rectangular blocks in air-cooled electronic units”. J. Fluids Eng., 116(4), pp. 856-861.

[11] Chen, S., and Jendrzejczyk, J., 1987. "Fluid excitation forces acting on a square tube array". J. Fluids Eng., 109(4), p. 415423.

[12] Bejan, A., and Morega, A., 1993. "Optimal arrays of pin fins and plate fins in laminar forced convection". J. Heat Transfer, 115, pp. 75-81.

[13] Sara, O., 2003. "Performance analysis of rectangular ducts with staggered square pin fins". Energy Convers. Manage., 44, pp. 1787-1803.

[14] Jeng, T., and Tzeng, S., 2007. "Pressure drop and heat transfer of square pin-fin arrays in in-line and staggered arrangements". Int. J. Heat Mass Transf., 50, pp. 2364-2375.

[15] Ruhlich, I., and Quack, H., 1999. "Investigations on regenerative heat exchangers". Cryocoolers 10, edited by RG Ross Jr., pp. 265-274.

[16] Sparrow, E., and Grannis, V., 1991. "Pressure drop characteristics of heat exchangers consisting of arrays of diamond-shaped pin fins". Int. J. Heat Mass Transfer, 34, pp. 589-600.

[17] Chyu, M., Hsing, Y., and Natarajan, V., 1998. "Convective heat transfer of cubic fin arrays in a narrow channel”. J. Trubomach., 120, pp. 362-367. 
[18] Tanda, G., 2001. "Heat transfer and pressure drop in a rectangular channel with diamondshaped elements". Int. J. Heat Mass Transf., 44, pp. 3529-3541.

[19] Jeng, T., 2006. "Thermal performance of in-line diamond-shaped pin fins in a rectangular duct”. Int. Commun. Heat Mass Transf., 33, pp. 1139-1146.

[20] Vanapalli, S., Ter Brake, H., Jansen, H., Burger, J., Holland, H., Veenstra, T., and Elwenspoek, M., 2007. "Pressure drop of laminar gas flows in a microchannel containing various pillar matrices". J. Micromech. Microeng., 17, pp. 1381-1386.

[21] Rasouli, E., and Narayanan, V., 2016. "Single-phase cryogenic flow and heat transfer through microscale pin fin heat sinks". Heat Transf. Eng., 37(11), pp. 994-1011.

[22] Grannis, V., and Sparrow, E., 1991. "Numerical simulation of fluid flow through an array of diamond-shaped pin fins”. Numer. Heat Tr. A-Appl., 19(4), pp. 381-403.

[23] Umeda, S., and Torii, S., 2012. "Numerical and experimental study on thermal fuild flow over twin diamond-shaped cylinders in free stream". 15th International Symposium on Flow Visualization, Minsk, Belarus, June 25-28.

[24] Torii, S., and Umeda, S., 2013. "Flip-flop flow control inside streamwise diverging diamondshaped cylinder bundles with concavities". J. Flow Control, Measurement Visualization, 1, pp. 77-85.

[25] Hirasawa, S., Fujiwara, A., Kawanami, T., and Shirai, K., 2014. "Forced convection heat transfer coefficient and pressure drop of diamond-shaped fin-array". J. Electronics Cooling Thermal Control, 4, pp. 78-85.

[26] De Smet, J., Gzil, P., Vervoort, N., Verelst, H., Baron, G., and Desmet, G., 2004. "Influence of the pillar shape on the band broadening and the separation impedance of perfectly ordered 2-d porous chromatographic media". Anal. Chem., 76, p. 3716.

[27] De Smet, J., Gzil, P., Vervoort, N., Verelst, H., Baron, G., and Desmet, G., 2005. "On the optimisation of the bed porosity and the particle shape of ordered chromatographic separation media". J. Chromatogr. A, 1073, pp. 43-51.

[28] De Pra, M., De Malsche, W., Desmet, G., Schoenmakers, P., and Kok, W., 2004. "Pillarstructured microchannels for on-chip liquid chromatography: Evaluation of the permeability 
and separation performance". J. Sep. Sci., 30, p. 1453.

[29] Rashidi, S., Bovand, M., Pop, I., and Valipour, M., 2014. "Numerical simulation of forced convective heat transfer past a square diamond-shaped porous cylinder". Transp. Porous Med., 102, pp. 207-225.

[30] Kays, W., and London, A., 1964. "Compact heat exchangers". New York: McGraw-Hill, p. chapters 1 and 7 .

[31] Gaddis, E., and Gnielski, V., 1985. "Pressure drop in horizontal cross flow across tube bundles". Int. Chem. Eng., 25(1), pp. 1-15.

[32] Kosar, A., Mishra, C., and Peles, Y., 2005. "Laminar flow across a bank of low aspect ratio micro pin fins". J. Fluids Eng., 19(4), p. 419.

[33] Kosar, A., Schneider, B., and Peles, Y., 2011. "Hydrodynamic characteristics of crossflow over mems-based pillars”. J. Fluids Eng., 133, pp. 081201-1.

[34] Tullius, J., Tullius, T., and Bayazitoglu, Y., 2012. "Optimization of short micro pin fins in minichannels". Int. J. Heat Mass Transf., 55, pp. 3921-3932.

[35] Rueda Villegas, L., Colombet, D., Guiraud, P., Legendre, D., Cazin, S., and Cockx, A., 2019. "Image processing for the experimental investigation of dense dispersed flows : Application to bubbly flows". Int. J. Multiph. Flow, 11, pp. 16-30.

[36] Petersen, H., 1970. "The properties of helium: Density, specific heats, viscosity, and thermal conductivity at pressures from 1 to 100 bar and from room temperature to about $1800 \mathrm{k}$. Roskilde, Denmark: Riso National Laboratory. Denmark. Forskningscenter Risoe. Risoe-R, 224.

[37] Colin, S., 2005. "Rarefaction and compressibility effects on steady or transient gas flows in microchannels". Microfluid. Nanofluid., 1, pp. 268-279.

[38] Mossaz, S., Colombet, D., Ledoux, G., and Ayela, F., 2015. "Role of the thermal entrance length on the viscous heating in microchannels". Microfluid. Nanofluid., 19, pp. 1325-1333.

[39] Gedeon, D., and Wood, J., 1996. "Oscillating-flow regenerator test rig: hadware and theory with derived correlations for sreens and felts". NASA CR-198442.

[40] Costa, S., Tutar, M., Barreno, I., Esnaola, J., Barrutia, H., Garcia, D., Gonzalez, M., and 
Prieto, J., 2014. "Experimental and numerical flow investigation of stirling engine regenerator". Energy, 72, pp. 800-812.

[41] Costa, S., Barrutia, H., Esnaola, J., and Tutar, M., 2013. "Numerical study of the pressure drop phenomena in wound woven wire matrix of a stirling regenerator". Energy Convers. Manag., 67, pp. 57-65.

[42] Ergun, S., 1952. "Fluid flow through packed columns". Chem. Engr. Prog., 48, pp. 89-94.

[43] Sochinskii, A., 2018. "First step toward the miniaturisation of space cryocoolers". PhD Thesis, Grenoble Alpes University.

[44] Vaschy, A., 1892. "Sur les lois de similitude en physique". Annales télégraphiques, 19, pp. 25-28.

[45] Buckingham, E., 1914. "On physically similar systems, illustration of the use of dimensional equations". Phys. Rev. 4, 4, pp. 345-376.

[46] Weller, H., Tabor, G., Jasak, H., and Fureby, C., 1998. "A tensorial approach to computational continuum mechanics using object-oriented techniques”. Comput. phys., 12, p. 620.

[47] Huang, H., Wu, H., and Zhang, C., 2018. "An experimental study on flow friction and heat transfer of water in sinusoidal wavy silicon microchannels". J. Micromech. Microeng., 28, p. 055003.

[48] Bird, R., Stewart, W., and Lightfood, E., 2002. "Transport phenomena, 2nd ed.”. John Wiley and sons, inc.

[49] Mulholland, J., Srivastava, R., and Wendt, J., 1988. "Influence of droplet spacing on drag coefficient in nonevaporating, monodisperse streams". AIAA Journal, 26, pp. 1231-1237.

[50] Kim, I., Elghobashi, S., and Sirignano, W., 1993. "Three-dimensional flow over two spheres placed side by side". J. Fluid. Mech., 246, pp. 465-488.

[51] Thrift, A., Brumbaugh, S., Thole, K., and Kohli, A., 2010. "A methodology to measure aerodynamic forces on cylinders in channel flow". J. Fluids Eng., 132(8), p. 08140.

[52] Sochinskii, A., Colombet, D., Medrano Muñoz, M., Ayela, F., and Luchier, N., 2019. "Flow and heat transfer around a diamond-shaped cylinder at moderate reynolds number". Int. J. Heat Mass Transf., 142, p. 118435. 
[53] Wen, L., and Yu, Y., 1966. "Mechanics of fluidization”. Chem. Engng. Prog. Symp. Ser., 62, pp. $100-111$.

[54] Larson, R., and Higdon, J., 1989. "A periodic grain consolidation model of porous media". Phys. fluids A, 1, p. 38.

[55] Van der Hoef, A., Beetstra, R., and Kuipers, J., 2005. "Lattice-boltzmann simulations of low-reynolds-number flow past mono- and bidisperse arrays of spheres: results for the permeability and drag force". J. Fluid Mech., 528, pp. 233-254.

[56] Sangani, A., and Acrivos, A., 1982. "Slow flow through a periodic array of spheres". Int. J. Multiph. Flow, 8(4), pp. 343-360.

[57] Stokes, G., 1851. "For creeping flow around an object of arbitrary shape". Trans. Cambridge Philos. Soc., 9 (part 2), p. 8. 


\section{LIST OF TABLES}

1 Geometrical parameters of the samples fabricated and studied in this work and the corresponding experimental Poiseuille number. Sizes are in micron, angle in degree. The measurements of $a, b, L_{L}, L_{T}$ from statistic averages from image treatment, * values calculated ( $D_{h}$ using Eq. 2, $\mathrm{t}=$ transparent Py/Si/Py samples) . . 41

2 Dimensions of the problem parameters: L: length, T: time, M: mass . . . . . . . . 42

3 The grid dimensions of the flow simulations in the cylinders array $\left(N_{e}\right.$ number of cells on $\Delta y, N_{c}$ number of cells along one 'c' segment, $N_{\text {total }}$ total number of cells) . 43

4 Poiseuille numbers obtained from numerical simulations for the case of a flow through a diamond-shaped cylinders matrix for $1 \leqslant R e \leqslant 30 \ldots \ldots$. . . . . . . . 44

5 Statistic about obtained Poiseuille number $\mathrm{Po}^{\prime}$ : average value $<P o^{\prime}>$, standard deviation $S T D\left(P o^{\prime}\right)$ and maximal absolute difference $\Delta \operatorname{Max}\left(\left|P o^{\prime}-<P o^{\prime}>\right|\right)$ compared to this value . . . . . . . . . . . . . . . . . . 45 


\section{LIST OF FIGURES}

1 (a) Schematic of a micro-channel with the micro structure region zoomed; (b) the picture of a Pyrex/Silicium/Pyrex sample . . . . . . . . . . . . . . . . . . 46

2 Geometrical parameters of the periodic diamond-shaped cylinders pattern considered in this work: (a) global parameters and (b) scheme for the calculation of the length $\Delta x$ imposed by the geometrical constraint $\Delta y=e \ldots \ldots \ldots \ldots$

3 SEM images of a sample: top view showing the porous region (a) border and (b) center with an incident angle of $30^{\circ}$; (c) front view used for the height measurements and showing vertical walls . . . . . . . . . . . . . . . . .

4 Example of optical microscope visualisations at same magnification factor $(\times 20$, images size $562 \times 421 \mu \mathrm{m}$ ), (e) example of image treatment for the measurements of sample dimensions with bounding box (in green) and centroid (in red), letter are related to the samples in Tab. $1 \ldots \ldots \ldots \ldots \ldots$

5 (a) Experimental set-up used for pressure drop measurements and (b) picture of the sample support . . . . . . . . . . . . . . . . . . . . . . . 50

6 Friction factor versus Reynolds number for three empty $2 \mathrm{~mm}$ width test channels of different height (without porous region): $\Delta h=17.2 \mu \mathrm{m} ; \bullet h=22.4 \mu \mathrm{m}$; $h=31.4 \mu \mathrm{m}$. The experimental data are compared to the theoretical friction factor for two parallel plates $-f=96 / R e \ldots \ldots \ldots \ldots \ldots$

7 Experimental friction factor versus Reynolds number for five samples with different porosity with $e \approx 20 \mu m$ for $\alpha=33^{\circ}: * \mathrm{~B} \varepsilon=0.46, \square \mathrm{C} \varepsilon=0.57, \Delta \mathrm{D} \varepsilon=0.65$ and for $\alpha=34^{\circ}: \bullet \mathrm{A} \varepsilon=0.45,, \nabla \mathrm{E} \varepsilon=0.68 \ldots \ldots \ldots \ldots$

8 Poiseuille number as a function of the matrix porosity: * experimental Poiseuille number for the 11 samples with $\alpha \approx 33^{\circ}$; Numerical simulation results for $\circ \alpha=33^{\circ}$, $\triangle \alpha=40^{\circ},{ }^{*} \alpha=50^{\circ}, \nabla \alpha=60^{\circ}$ and $\square \alpha=90^{\circ} ;---$ estimation of the Poiseuille number from the parallel-plates flow analogy $\left(P o=<P o^{\prime}>\left(D_{h} / D_{h}^{\prime}\right)^{2}\left(L_{L}^{\prime} / L_{L}\right)\right.$ and Eq. 24); ... stacked spheres matrices $P o=133$ [42]; ... stacked woven wire matrices $P o=123$ [41]; -.- flow between two parallel plates $P o=96 \ldots \ldots$. . . . . . 53 
9 (a) Computational domain and (b) example of a coarse mesh for $\alpha=33^{\circ}$ and $\varepsilon=$ $60 \%$ (here $N_{\text {total }}=1792, N_{e}=16$ and $N_{c}=24$ against $N_{\text {total }}=35200, N_{e}=100$ and $N_{c}=83$ for the mesh used for the numerical simulation, see Tab.3) $\ldots \ldots \ldots$

10 Velocity field in a periodic domain for $\varepsilon=40 \%$ with (a) $\alpha=33^{\circ}$, (b) $\alpha=60^{\circ}$ and (c) $\alpha=90^{\circ}$ at $R e=1$ (left), $R e=30$ (right) . . . . . . . . . . . . . . . 55

11 Velocity field in a periodic domain for $\varepsilon=80 \%$ with (a) $\alpha=33^{\circ}$, (b) $\alpha=60^{\circ}$ and (c) $\alpha=90^{\circ}$ at $R e=1$ (left), $R e=30$ (right) . . . . . . . . . . . . . . 56

12 Friction factor as a function of the exchanger Reynolds number $\operatorname{Re}$ for (a) $\alpha=33^{\circ}$ and (b) $\alpha=90^{\circ}$ with $\circ \varepsilon=0.40, \circ \varepsilon=0.50, \circ \varepsilon=0.60, \circ \varepsilon=0.70, \circ \varepsilon=0.80$, $\varepsilon=0.90 ;-$ - flow between two parallel plates $f=96 / R e \ldots \ldots$. . . . . . . 57

13 (a) Tortuosity and hydraulic diameters ratio as a function of the porosity for : o $\alpha=33^{\circ}, \triangle \alpha=40^{\circ},{ }^{*} \alpha=50^{\circ}, \nabla \alpha=60^{\circ}$ et $\square \alpha=90^{\circ} \ldots \ldots \ldots \ldots$

14 Parallel-plates flow analogy (a) Poiseuille number $P o^{\prime}$ as a function of the porosity for: $\circ \alpha=33^{\circ}, \triangle \alpha=40^{\circ},{ }^{*} \alpha=50^{\circ}, \nabla \alpha=60^{\circ}$ et $\square \alpha=90^{\circ}$; -.- flow between two parallel plates $P o^{\prime}=96$; (b) Corresponding average Poiseuille numbers $<P o^{\prime}>$ as a function of the apex angle: $\circ$ numerical simulations and - Eq. $24 \ldots \ldots$

15 Drag coefficient as a function of the cylinder Reynolds number $\operatorname{Re}_{D}$ : (a) $\alpha=33^{\circ}$ (b) $\alpha=90^{\circ}$ and $\circ \varepsilon=0.40, \circ \varepsilon=0.50, \circ \varepsilon=0.60, \circ \varepsilon=0.70, \circ \varepsilon=0.80, \circ \varepsilon=0.90$; $---C_{D}$ drag coefficient for a single cylinder according to Sochinskii et al. [52]; .... estimation of the drag coefficient using $\left\langle P o^{\prime}\right\rangle$ Eq. 24 and $25 \ldots \ldots$. . . . . . . . 60 
16 Poiseuille number comparison to the circular cylinders and spherical particles array cases: square array of circular cylinders according to $\nabla$ numerical simulations of Sangani \& Acrivos [2] and $\triangle$ experiments of Yeom et al. [6]; $\triangleright$ staggered array of circular cylinders according to numerical simulations of Sangani \& Acrivos [2]; * simple cubic array of spheres, + body centered cubic array of spheres, $\times$ face centered cubic array of spheres according to numerical simulations of Sangani \& Acrivos [56]; present numerical simulations with diamond-shaped cylinders arrays for $\circ \alpha=33^{\circ}$ and $\square \alpha=90^{\circ}$; --- estimation of the Poiseuille number applying the parallel-plates flow analogy $\left(P o=<P o^{\prime}>\left(D_{h} / D_{h}^{\prime}\right)^{2}\left(L_{L}^{\prime} / L_{L}\right)\right.$ and Eq. 24); -- flow between two parallel plates $P_{o}=96$; asymptotic behaviour for very dilute - spheres arrays and diamond-shaped cylinders arrays with $-\alpha=33^{\circ}$ and $\alpha=90^{\circ}($ Eq. 27) . . . . . . . . . . . . . . . . . . . . . 61 
Table 1. Geometrical parameters of the samples fabricated and studied in this work and the corresponding experimental Poiseuille number. Sizes are in micron, angle in degree. The measurements of $a, b, L_{L}, L_{T}$ from statistic averages from image treatment, * values calculated ( $D_{h}$ using Eq. $2, \mathrm{t}=$ transparent Py/Si/Py samples)

\begin{tabular}{c|c|c|c|c|c|c|c|c|c|c|c|c}
\hline sample & $\varepsilon^{*}$ & $e^{*}$ & $\alpha$ & $a$ & $b$ & $c^{*}$ & $L_{L}$ & $L_{T}$ & $D_{h}^{*}$ & $\mathrm{H}$ & $\frac{A_{\text {cont }}^{w}}{A_{\text {cont }}}$ & Po \\
\hline$A^{t}$ & 0.45 & 20.2 & 34 & 65.8 & 218.9 & 114.3 & 245.7 & 106.2 & 46.0 & 230 & 0.11 & 113 \\
$B$ & 0.46 & 20.95 & 33 & 64.4 & 217.6 & 113.5 & 246.1 & 106.3 & 45.6 & 154 & 0.17 & 96 \\
$C$ & 0.57 & 20.7 & 33 & 41.7 & 139.6 & 72.8 & 161.7 & 83.1 & 42.9 & 120 & 0.22 & 104 \\
$D^{t}$ & 0.65 & 20.25 & 33 & 30.0 & 96.4 & 50.5 & 117.9 & 70.5 & 48.3 & 240 & 0.11 & 149 \\
$E^{t}$ & 0.68 & 21.6 & 34 & 27.3 & 97.2 & 50.5 & 117.8 & 70.5 & 46.6 & 140 & 0.19 & 132 \\
$F^{t}$ & 0.64 & 40.65 & 32 & 59.6 & 202.4 & 105.5 & 235.1 & 140.9 & 74.1 & 144 & 0.35 & 138 \\
$G$ & 0.65 & 40.85 & 34 & 59.1 & 197.0 & 102.8 & 234.8 & 140.8 & 78.6 & 160 & 0.32 & 109 \\
$H^{t}$ & 0.50 & 10.5 & 34 & 32.1 & 101.8 & 53.4 & 122.3 & 53.1 & 28.2 & 212 & 0.07 & 113 \\
$I$ & 0.55 & 11.5 & 34 & 30.0 & 98.0 & 51.2 & 122.4 & 53.0 & 30.4 & 127 & 0.13 & 98 \\
$J$ & 0.70 & 10.9 & 33 & 13.4 & 45.5 & 23.7 & 57.8 & 35.2 & 26.4 & 108 & 0.13 & 119 \\
$K$ & 0.78 & 11.65 & 33 & 11.8 & 37.9 & 19.8 & 57.7 & 35.1 & 34.8 & 140 & 0.14 & 121 \\
\hline
\end{tabular}


Journal of Fluids Engineering

Table 2. Dimensions of the problem parameters: L: length, T: time, M: mass

\begin{tabular}{c|c|c|c|c|c|c|c|c}
\hline & $\Delta p$ & $\mathrm{U}$ & $D_{h}$ & $\rho$ & $\mu$ & $\mathrm{a}$ & $\mathrm{b}$ & $\mathrm{L}$ \\
\hline $\mathrm{L}$ & -1 & 1 & 1 & -3 & -1 & 1 & 1 & 1 \\
$\mathrm{~T}$ & -2 & -1 & 0 & 0 & -1 & 0 & 0 & 0 \\
$\mathrm{M}$ & 1 & 0 & 0 & 1 & 1 & 0 & 0 & 0 \\
\hline
\end{tabular}


Table 3. The grid dimensions of the flow simulations in the cylinders array ( $N_{e}$ number of cells on $\Delta y, N_{c}$ number of cells along one 'c' segment, $N_{\text {total }}$ total number of cells)

\begin{tabular}{c|c|c|c|c}
\hline$\alpha$ & $\varepsilon$ & $N_{\text {total }}$ & $N e$ & $N c$ \\
\hline 33 & 0.4 & 16400 & 50 & 80 \\
& 0.5 & 23520 & 70 & 81 \\
& 0.6 & 35200 & 100 & 83 \\
& 0.7 & 64800 & 180 & 82 \\
& 0.8 & 96000 & 250 & 84 \\
& 0.9 & 139200 & 300 & 90 \\
\hline 40 & 0.4 & 10800 & 50 & 52 \\
& 0.5 & 15680 & 70 & 53 \\
& 0.6 & 22400 & 100 & 52 \\
& 0.7 & 34800 & 150 & 52 \\
\hline 50 & 0.4 & 6800 & 50 & 32 \\
& 0.5 & 9520 & 70 & 31 \\
& 0.6 & 14400 & 100 & 32 \\
& 0.7 & 22800 & 150 & 32 \\
\hline 60 & 0.4 & 9600 & 40 & 55 \\
& 0.5 & 9920 & 40 & 56 \\
& 0.6 & 19600 & 70 & 60 \\
& 0.7 & 27200 & 100 & 54 \\
& 0.8 & 64800 & 180 & 65 \\
\hline 90 & 0.4 & 8400 & 50 & 36 \\
& 0.5 & 12320 & 70 & 36 \\
& 0.6 & 19200 & 100 & 36 \\
& 0.7 & 32400 & 150 & 36 \\
& 0.8 & 51200 & 200 & 36 \\
\hline
\end{tabular}


Journal of Fluids Engineering

Table 4. Poiseuille numbers obtained from numerical simulations for the case of a flow through a diamond-shaped cylinders matrix for $1 \leqslant R e \leqslant 30$

\begin{tabular}{|c|c|c|}
\hline$\alpha$ & $\varepsilon$ & $P o$ \\
\hline \multirow[t]{6}{*}{33} & 0.40 & 109.58 \\
\hline & 0.50 & 111.81 \\
\hline & 0.60 & 115.32 \\
\hline & 0.70 & 121.06 \\
\hline & 0.80 & 132.04 \\
\hline & 0.90 & 160.01 \\
\hline \multirow[t]{4}{*}{40} & 0.40 & 116.56 \\
\hline & 0.50 & 119.91 \\
\hline & 0.60 & 125.29 \\
\hline & 0.70 & 133.58 \\
\hline \multirow[t]{4}{*}{50} & 0.40 & 130.09 \\
\hline & 0.50 & 135.44 \\
\hline & 0.60 & 144.17 \\
\hline & 0.70 & 157.03 \\
\hline \multirow[t]{5}{*}{60} & 0.40 & 143.88 \\
\hline & 0.50 & 151.39 \\
\hline & 0.60 & 161.84 \\
\hline & 0.70 & 178.36 \\
\hline & 0.80 & 206.52 \\
\hline \multirow[t]{5}{*}{90} & 0.40 & 234.69 \\
\hline & 0.50 & 252.45 \\
\hline & 0.60 & 272.99 \\
\hline & 0.70 & 305.07 \\
\hline & 0.80 & 357.11 \\
\hline
\end{tabular}


Table 5. Statistic about obtained Poiseuille number $P o^{\prime}$ : average value $<P o^{\prime}>$, standard deviation $S T D\left(P o^{\prime}\right)$ and maximal absolute difference $\triangle M a x\left(\left|P o^{\prime}-<P o^{\prime}>\right|\right)$ compared to this value

\begin{tabular}{c|c|c|c}
\hline$\alpha$ & $<P o^{\prime}>$ & $S T D\left(P o^{\prime}\right)$ & $\Delta \operatorname{Max}\left(\left|P o^{\prime}-<P o^{\prime}>\right|\right)$ \\
\hline 33 & 98 & 1.74 & 3.15 \\
40 & 101.9 & 0.37 & 0.49 \\
50 & 106.7 & 0.27 & 0.35 \\
60 & 108.3 & 2.1 & 3.51 \\
90 & 137.3 & 4.3 & 7.07 \\
\hline
\end{tabular}




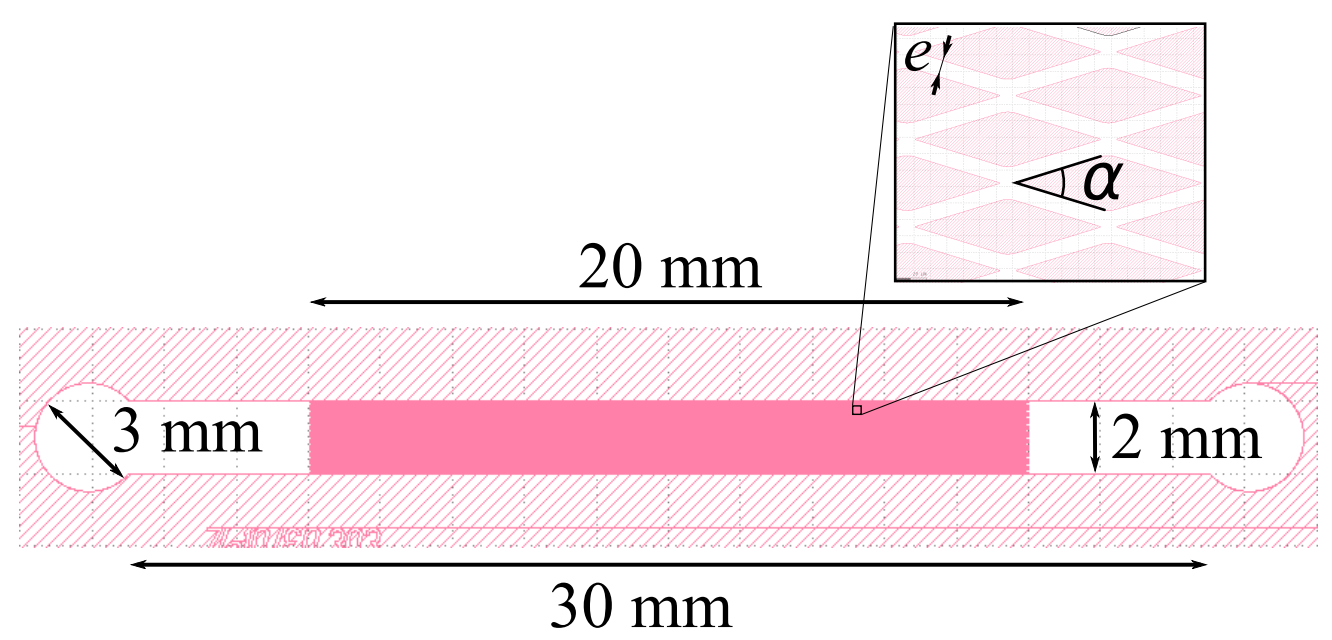

(a)

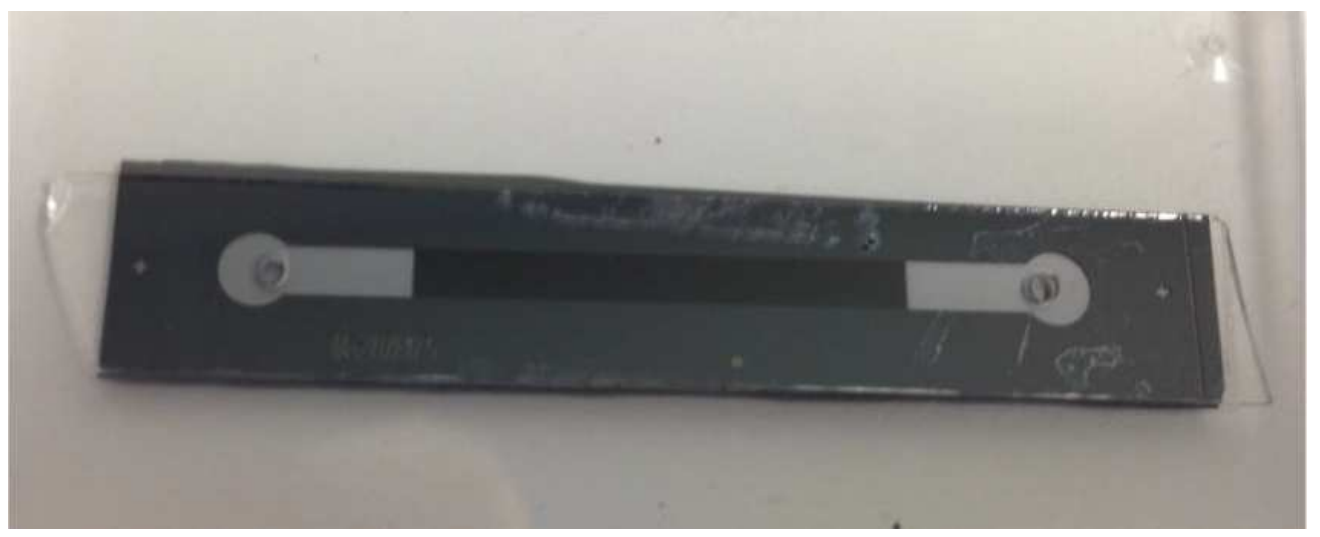

(b)

Fig. 1. (a) Schematic of a micro-channel with the micro structure region zoomed; (b) the picture of a Pyrex/Silicium/Pyrex sample 


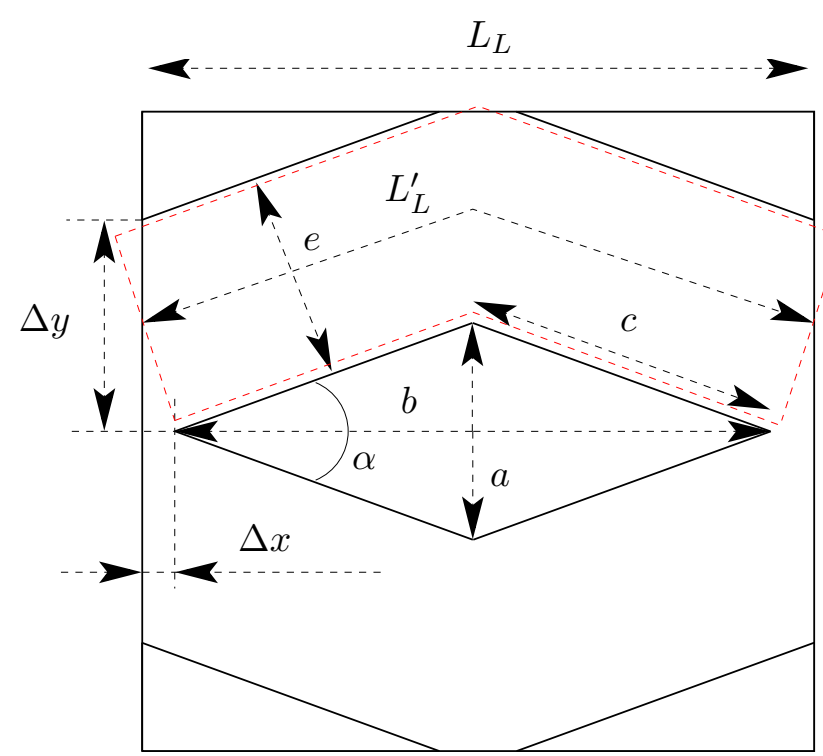

(a)

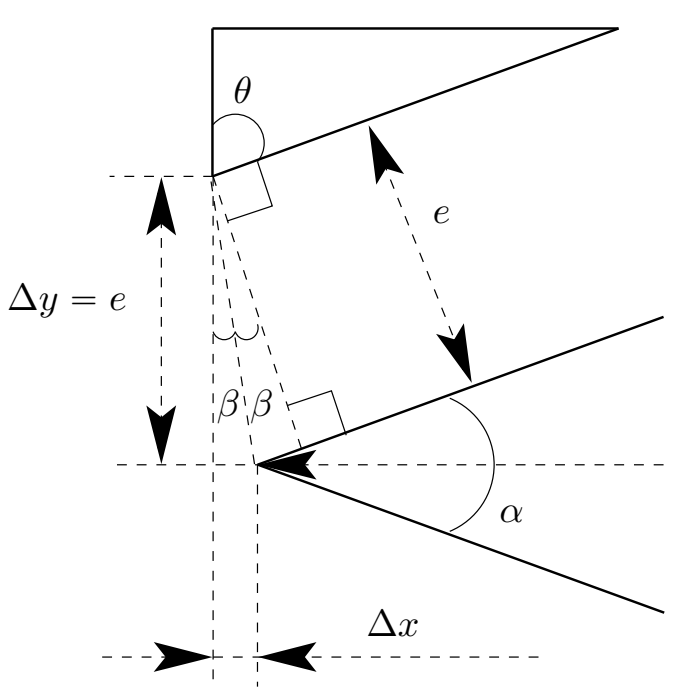

(b)

Fig. 2. Geometrical parameters of the periodic diamond-shaped cylinders pattern considered in this work: (a) global parameters and (b) scheme for the calculation of the length $\Delta x$ imposed by the geometrical constraint $\Delta y=e$ 
Journal of Fluids Engineering

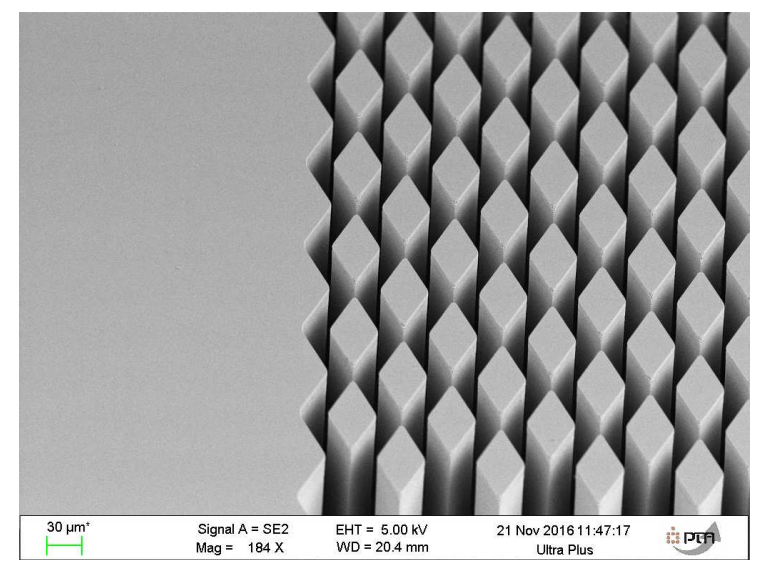

(a)

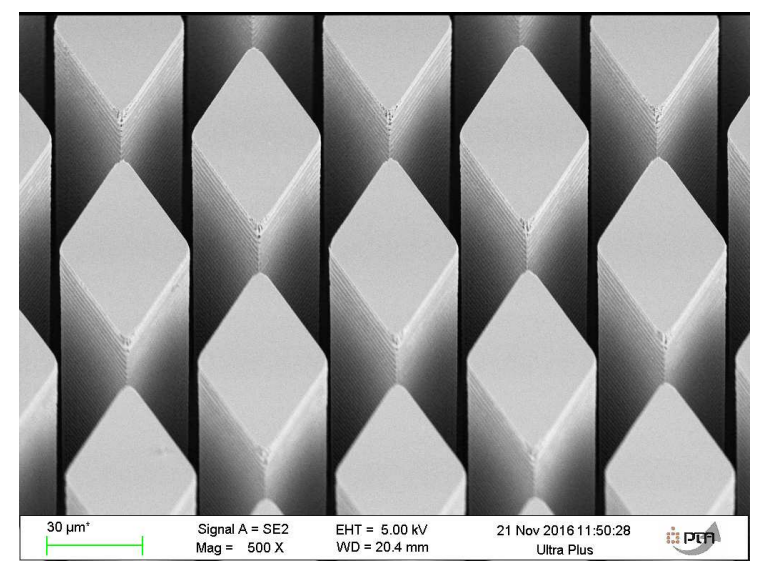

(b)

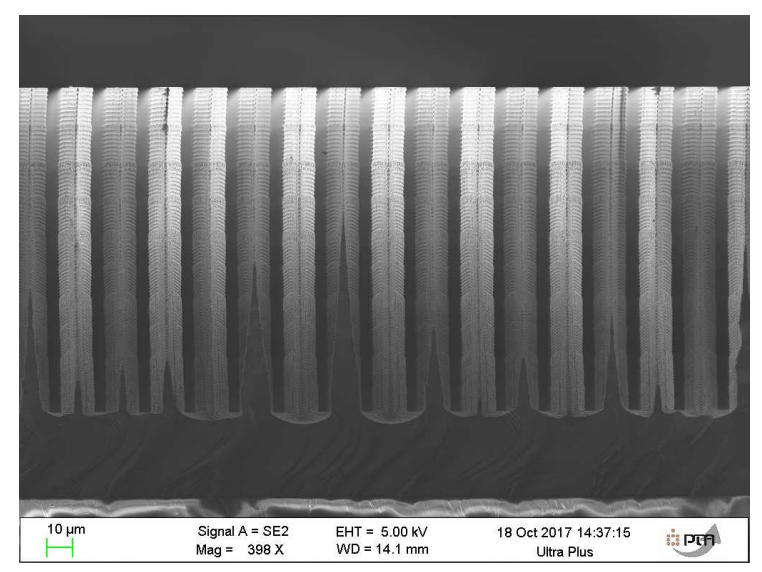

(c)

Fig. 3. SEM images of a sample: top view showing the porous region (a) border and (b) center with an incident angle of $30^{\circ}$; (c) front view used for the height measurements and showing vertical walls 


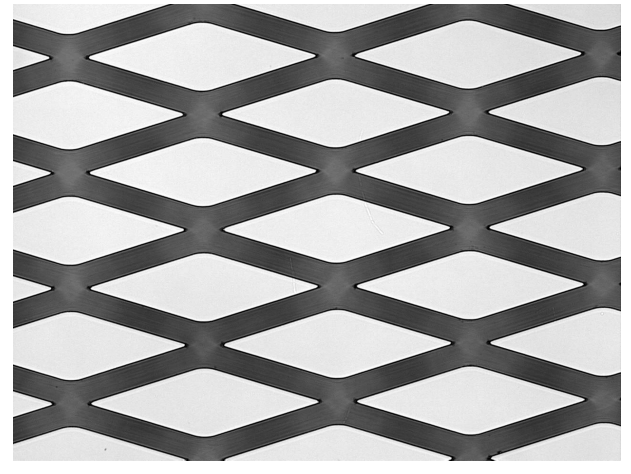

(a) $\mathrm{B}-\varepsilon=0.46 e=21 \mu m a=64.4 \mu m$

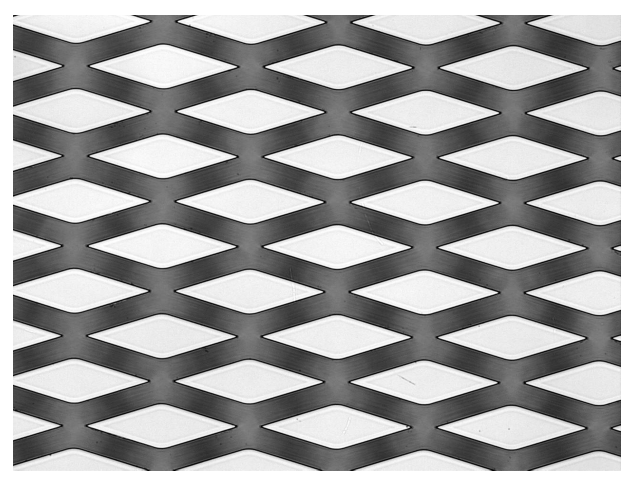

(c) $\mathrm{C}-\varepsilon=0.57 e=20.7 \mu m a=41.7 \mu m$

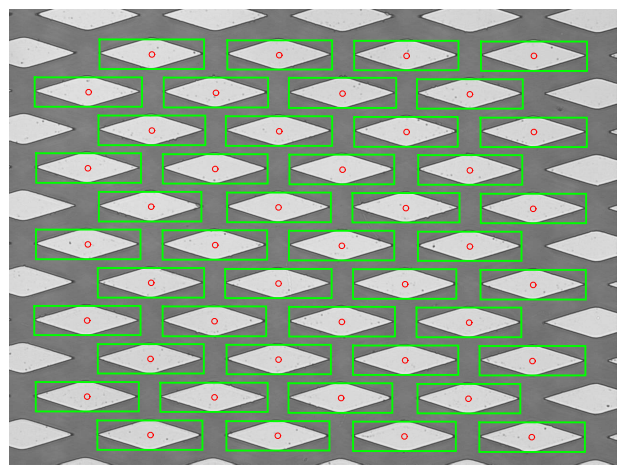

(e) $\mathrm{E}-\varepsilon=0.68 e=21.6 \mu \mathrm{m} a=27.3 \mu \mathrm{m}$

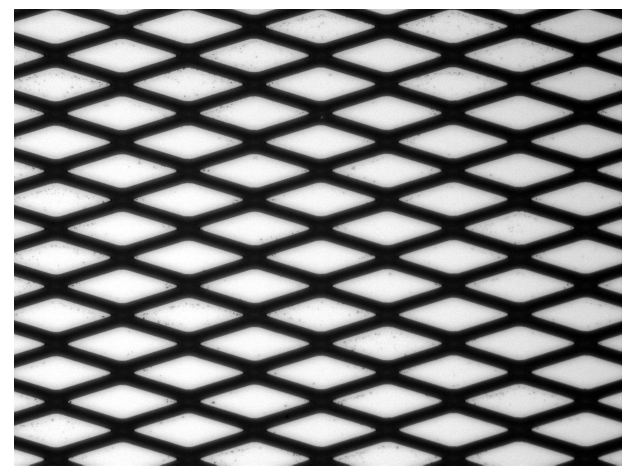

(b) $\mathrm{H}-\varepsilon=0.50 e=10.5 \mu m a=32.1 \mu m$

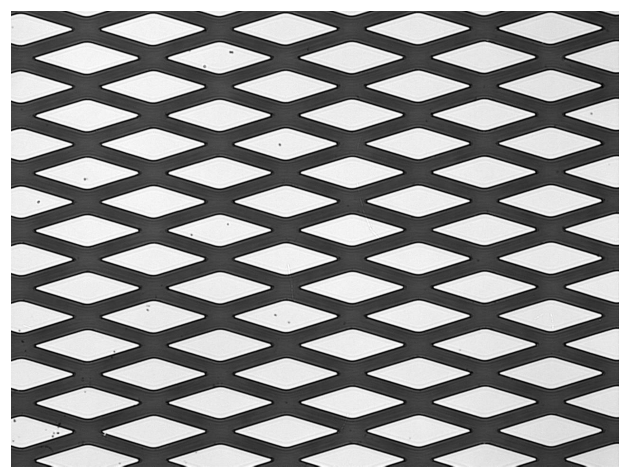

(d) I - $\varepsilon=0.55 e=11.5 \mu \mathrm{m} a=30 \mu \mathrm{m}$

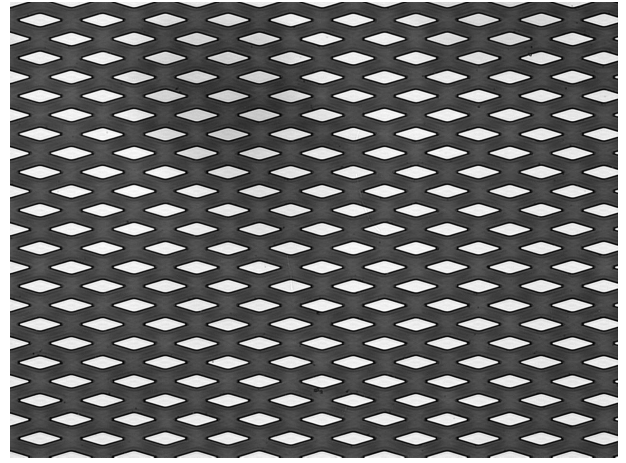

(f) $\mathrm{K}-\varepsilon=0.78 e=11.7 \mu \mathrm{m} a=11.8 \mu \mathrm{m}$

Fig. 4. Example of optical microscope visualisations at same magnification factor $(\times 20$, images size $562 \times 421 \mu m$ ), (e) example of image treatment for the measurements of sample dimensions with bounding box (in green) and centroid (in red), letter are related to the samples in Tab. 1 


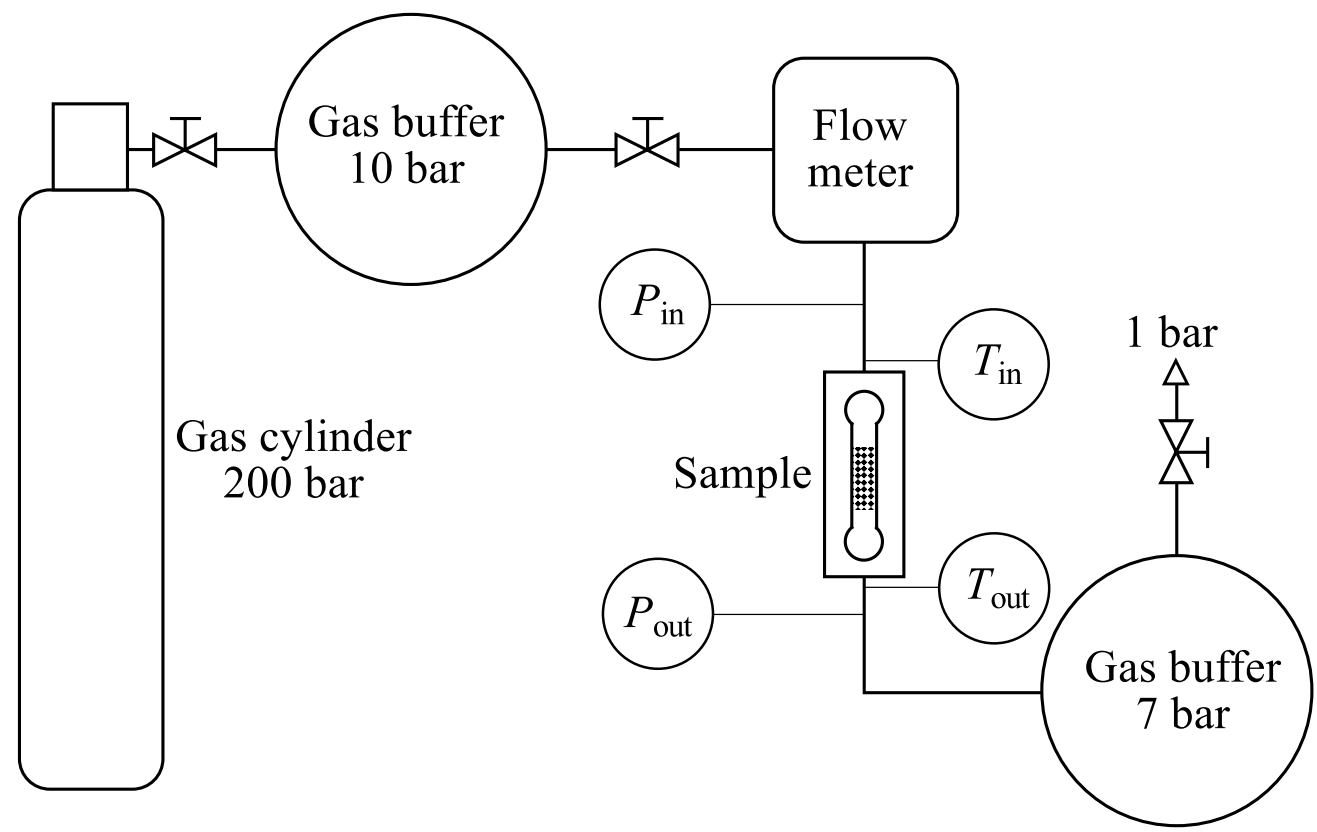

(a)

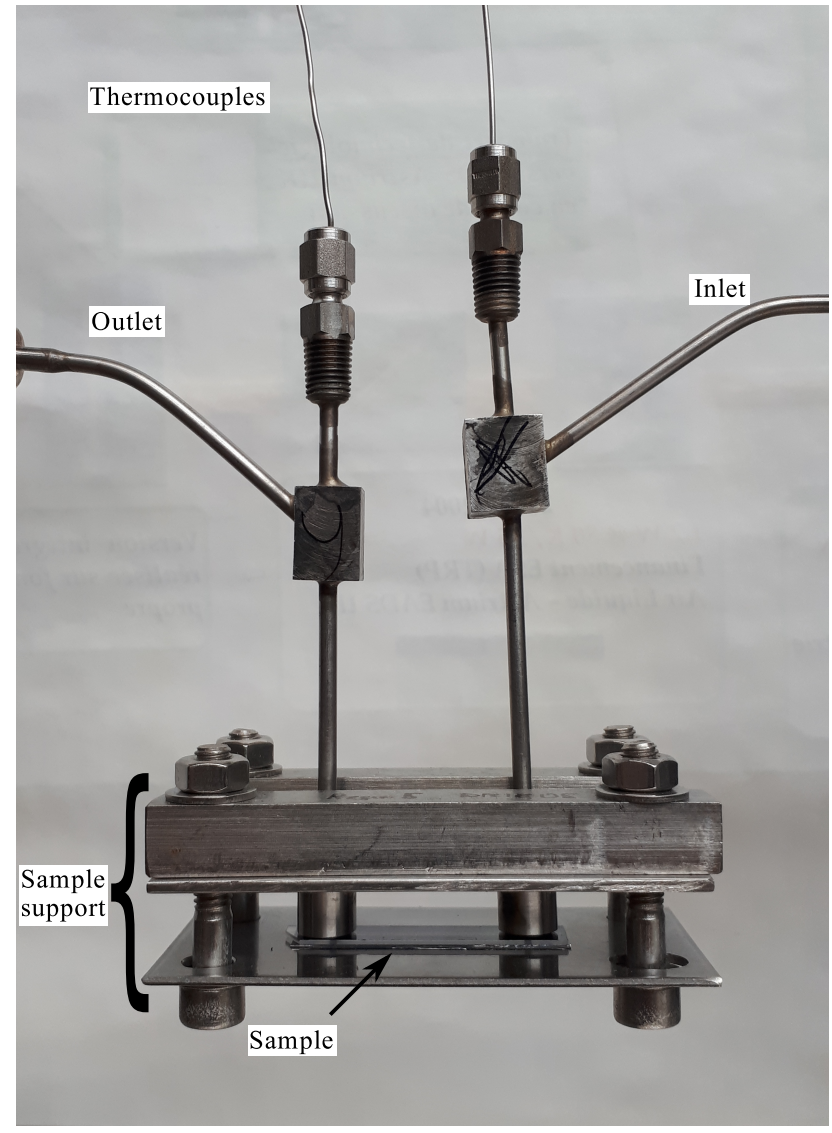

(b)

Fig. 5. (a) Experimental set-up used for pressure drop measurements and (b) picture of the sample support 


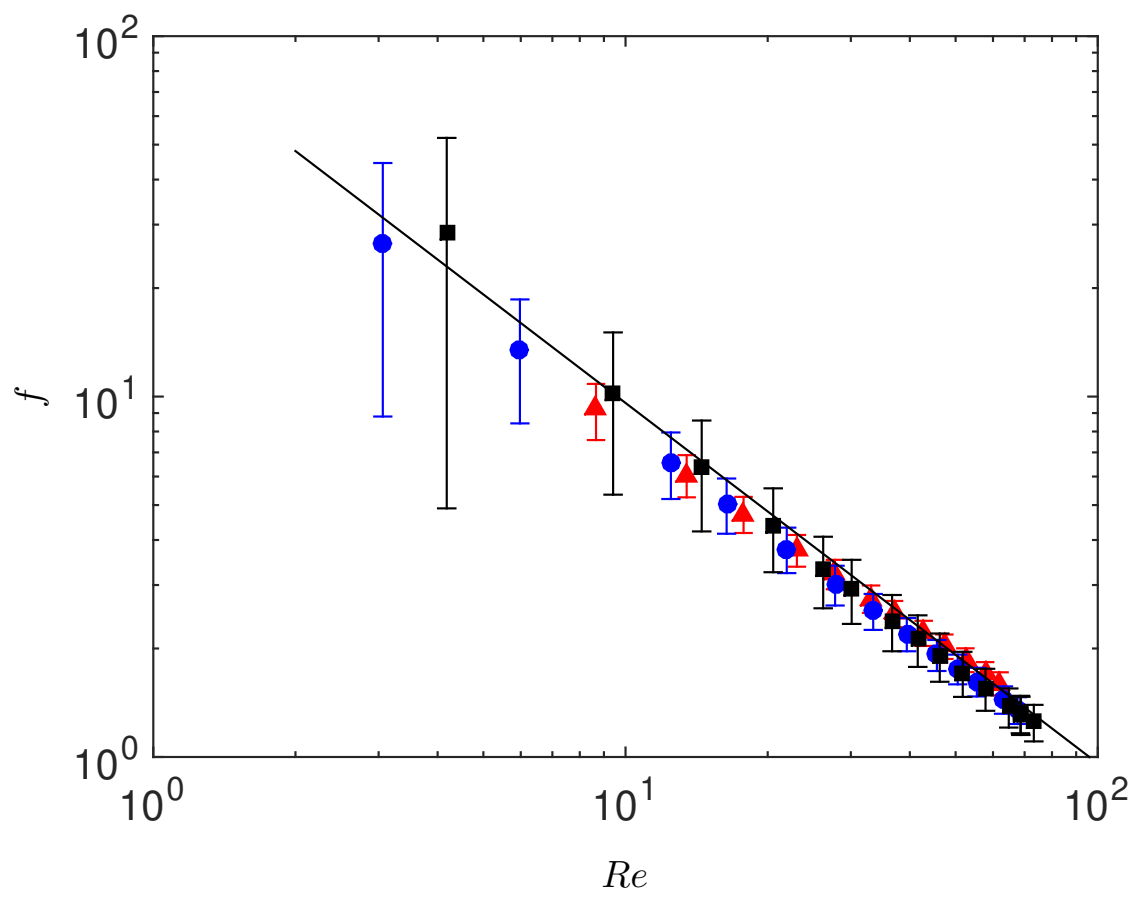

Fig. 6. Friction factor versus Reynolds number for three empty $2 \mathrm{~mm}$ width test channels of different height (without porous region): $\Delta h=17.2 \mu \mathrm{m} ; \bullet h=22.4 \mu \mathrm{m} ; \boldsymbol{\square}=31.4 \mu \mathrm{m}$. The experimental data are compared to the theoretical friction factor for two parallel plates $-f=96 / R e$ 


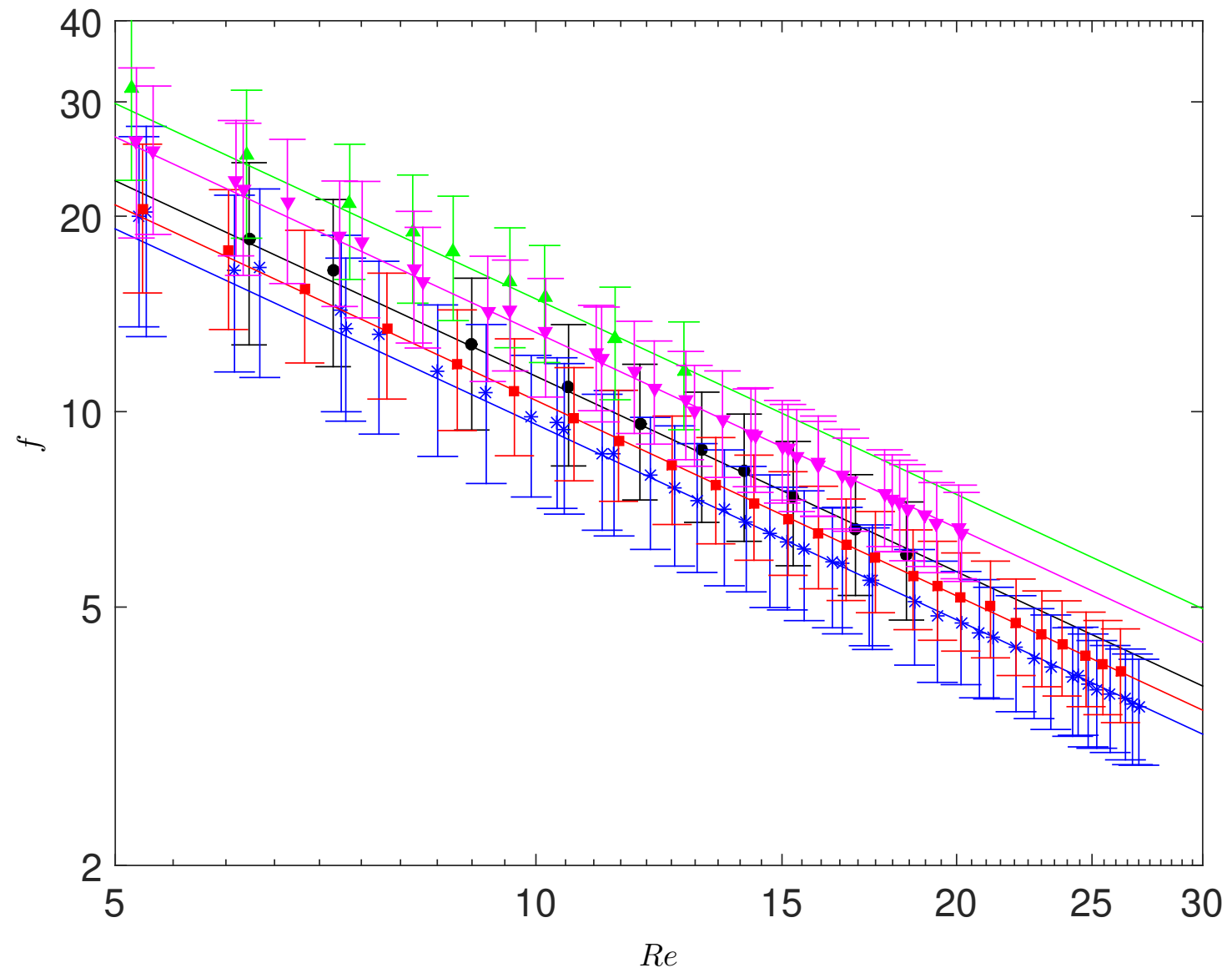

Fig. 7. Experimental friction factor versus Reynolds number for five samples with different porosity with $e \approx 20 \mu m$ for $\alpha=33^{\circ}$ : $* \mathrm{~B} \varepsilon=0.46, \square \mathrm{C} \varepsilon=0.57, \triangle \mathrm{D} \varepsilon=0.65$ and for $\alpha=34^{\circ}: \bullet \mathrm{A} \varepsilon=0.45$, , $\varepsilon \mathrm{E} \varepsilon=0.68$ 


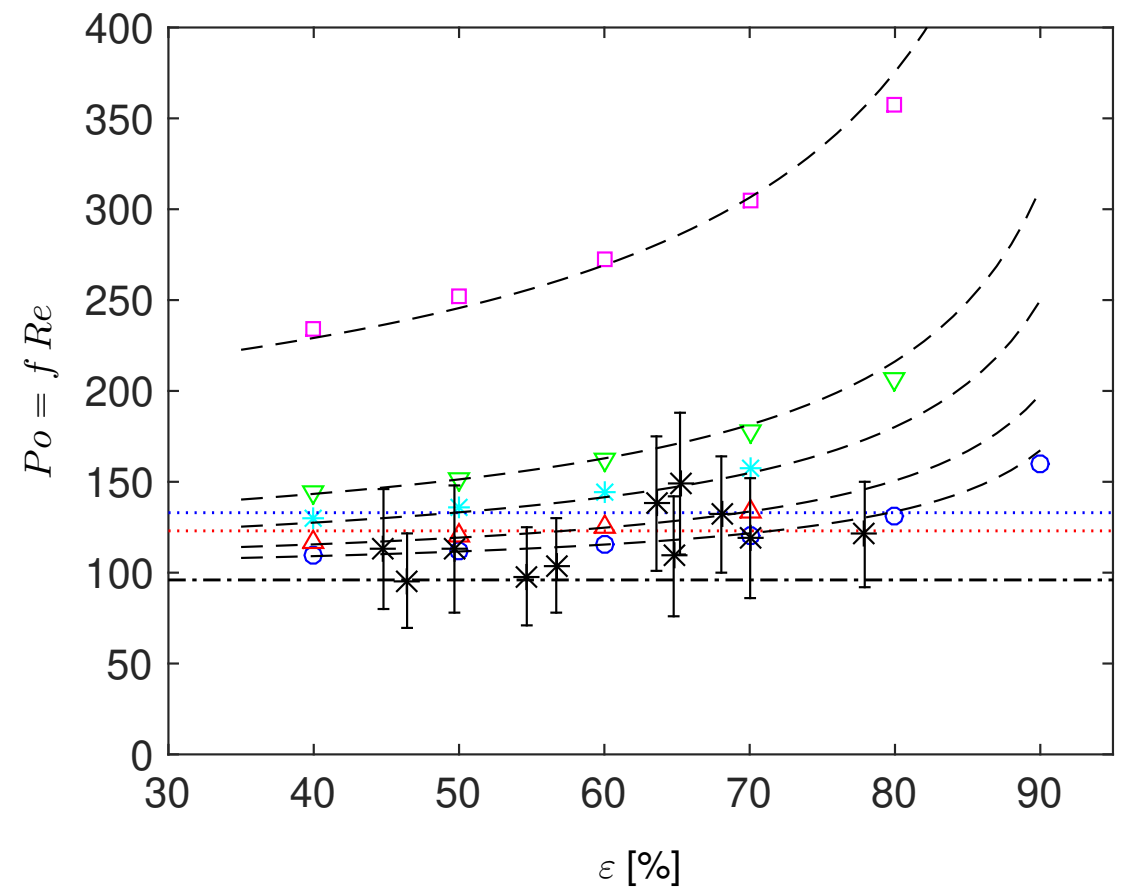

Fig. 8. Poiseuille number as a function of the matrix porosity: * experimental Poiseuille number for the 11 samples with $\alpha \approx 33^{\circ}$; Numerical simulation results for $\circ \alpha=33^{\circ}, \triangle \alpha=40^{\circ},{ }^{*} \alpha=50^{\circ}, \nabla \alpha=60^{\circ}$ and $\square \alpha=90^{\circ}$; -- estimation of the Poiseuille number from the parallel-plates flow analogy $\left(P o=<P o^{\prime}>\left(D_{h} / D_{h}^{\prime}\right)^{2}\left(L_{L}^{\prime} / L_{L}\right)\right.$ and Eq. 24); ... stacked spheres matrices $P o=133$ [42]; ... stacked woven wire matrices $P o=123$ [41]; -.- flow between two parallel plates $P o=96$ 


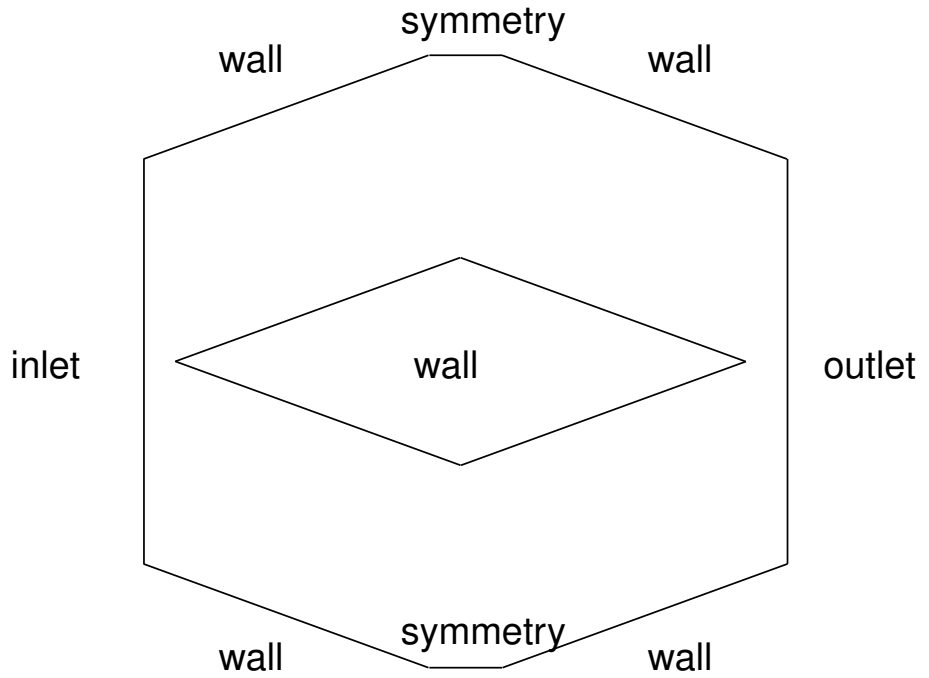

(a)

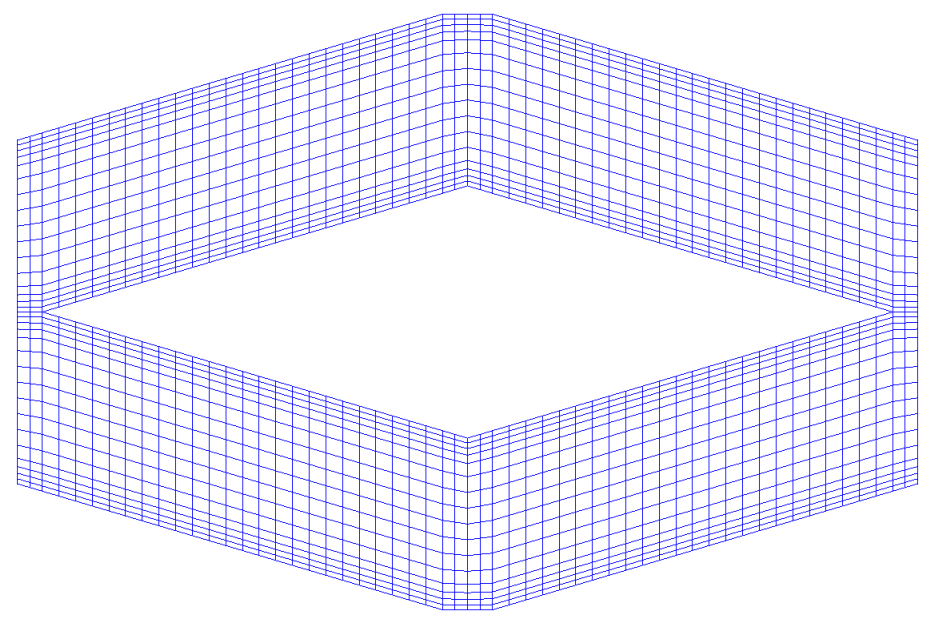

(b)

Fig. 9. (a) Computational domain and (b) example of a coarse mesh for $\alpha=33^{\circ}$ and $\varepsilon=60 \%$ (here $N_{\text {total }}=1792$, $N_{e}=16$ and $N_{c}=24$ against $N_{\text {total }}=35200, N_{e}=100$ and $N_{c}=83$ for the mesh used for the numerical simulation, see Tab.3) 
(a)
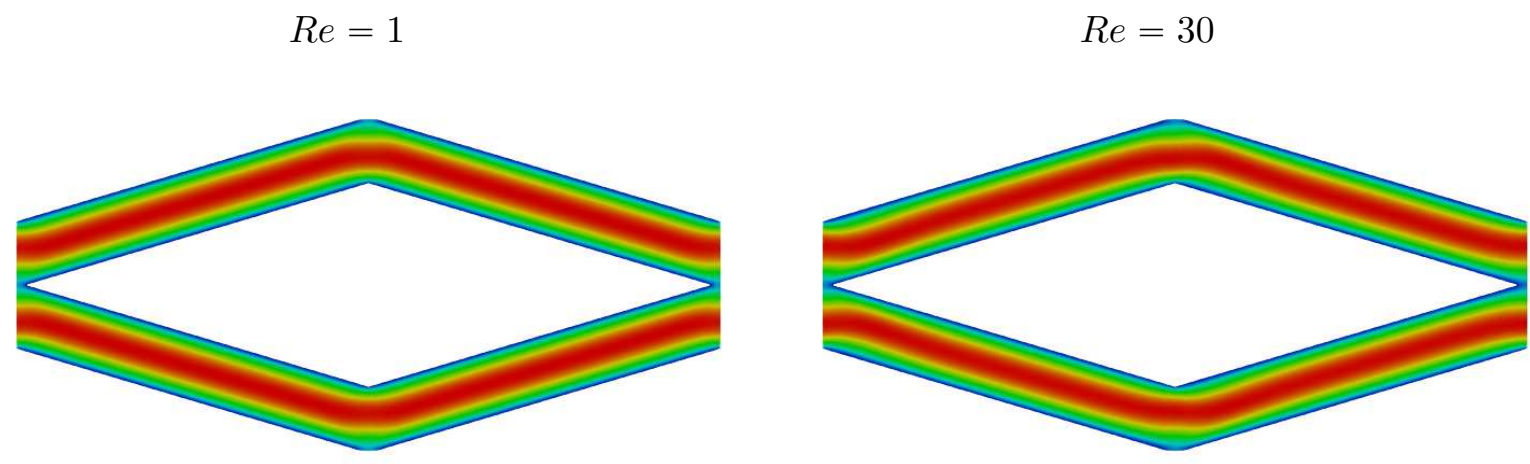

(b)
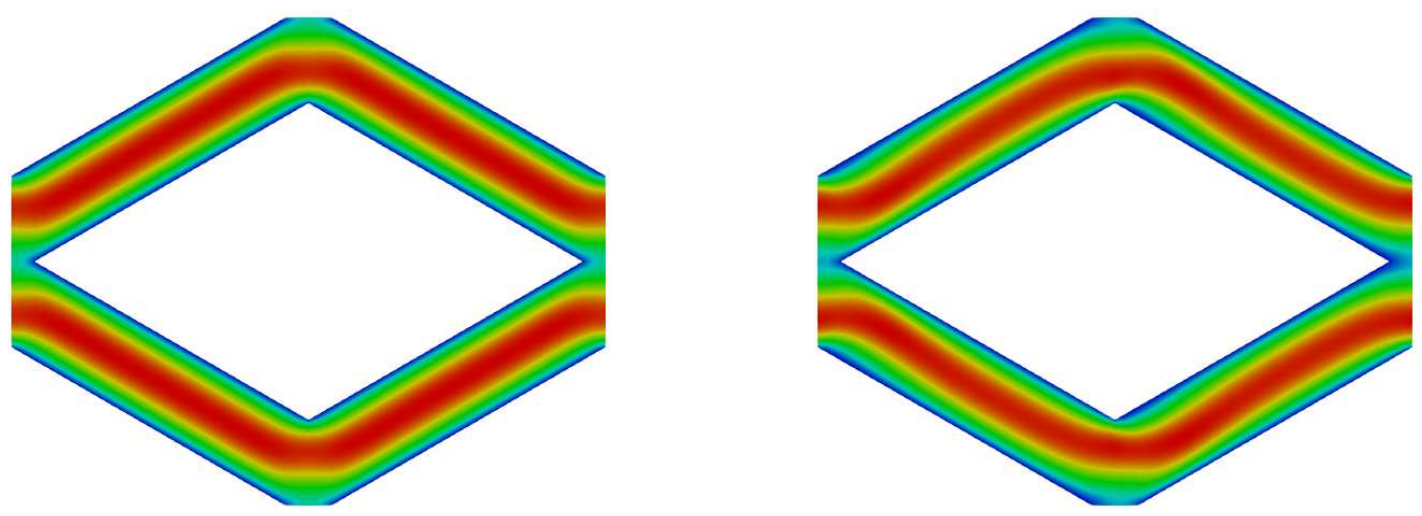

(c)
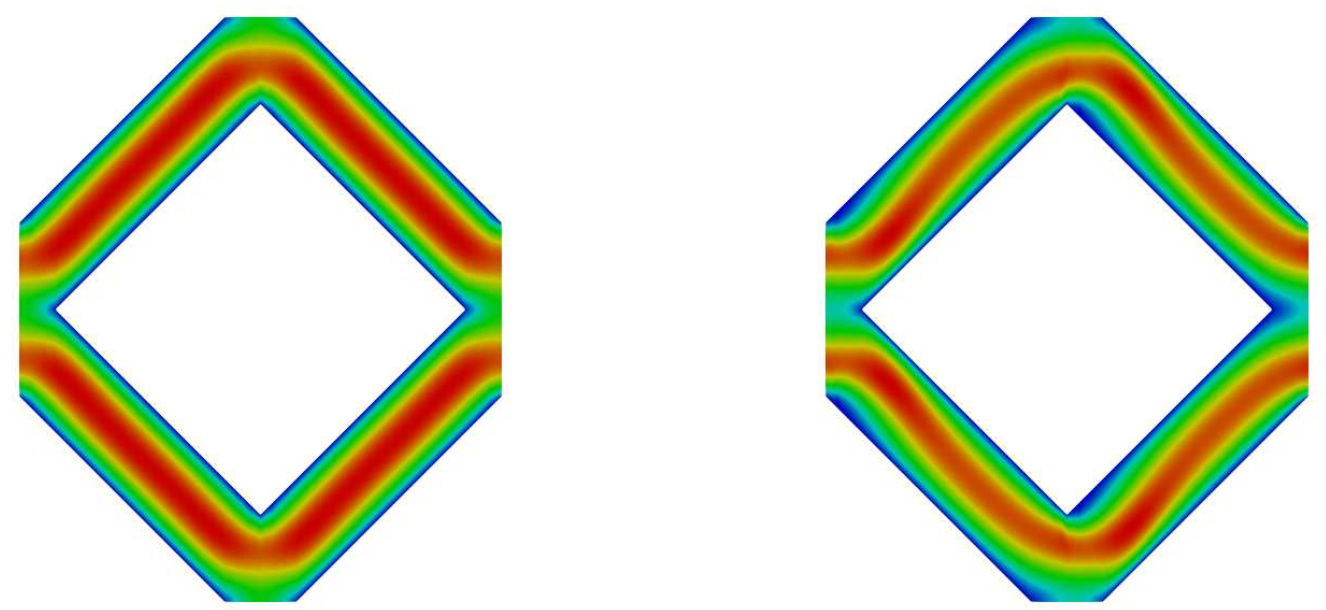

Fig. 10. Velocity field in a periodic domain for $\varepsilon=40 \%$ with (a) $\alpha=33^{\circ}$, (b) $\alpha=60^{\circ}$ and (c) $\alpha=90^{\circ}$ at $R e=1$ (left), $R e=30$ (right) 


\section{Journal of Fluids Engineering}

(a)
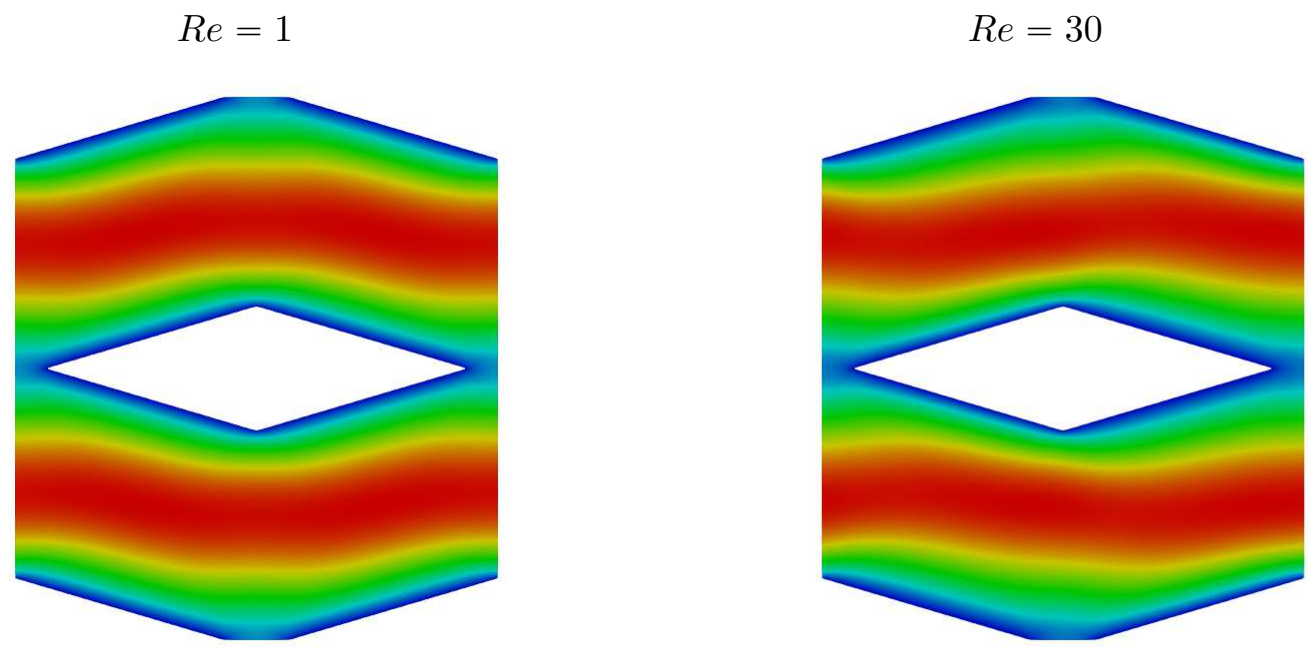

(b)
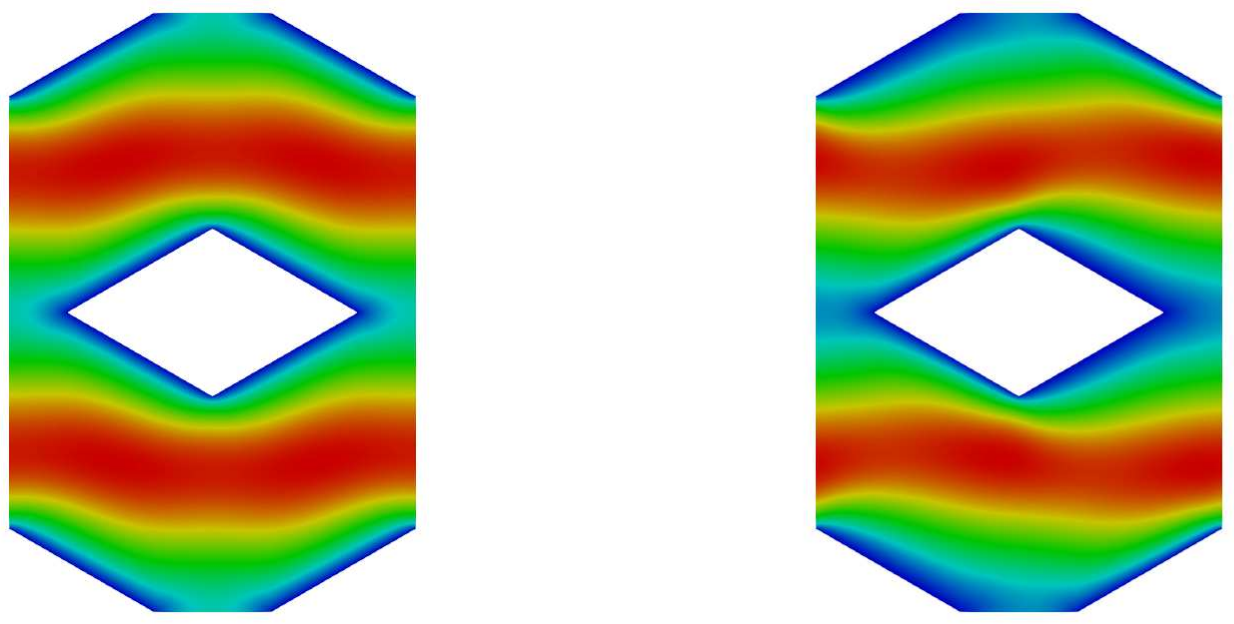

(c)
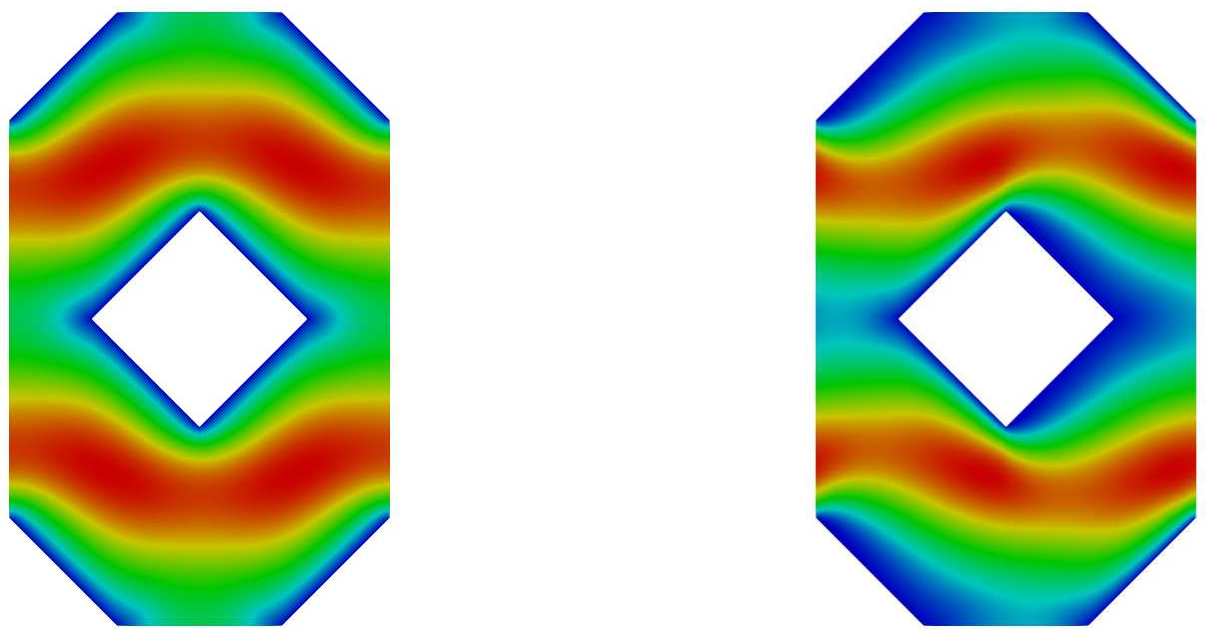

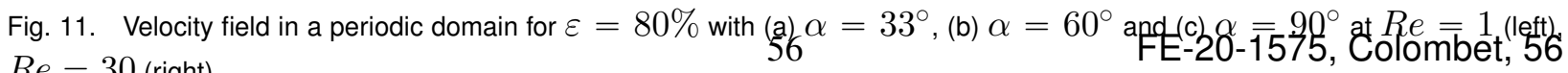
$R e=30$ (right) 


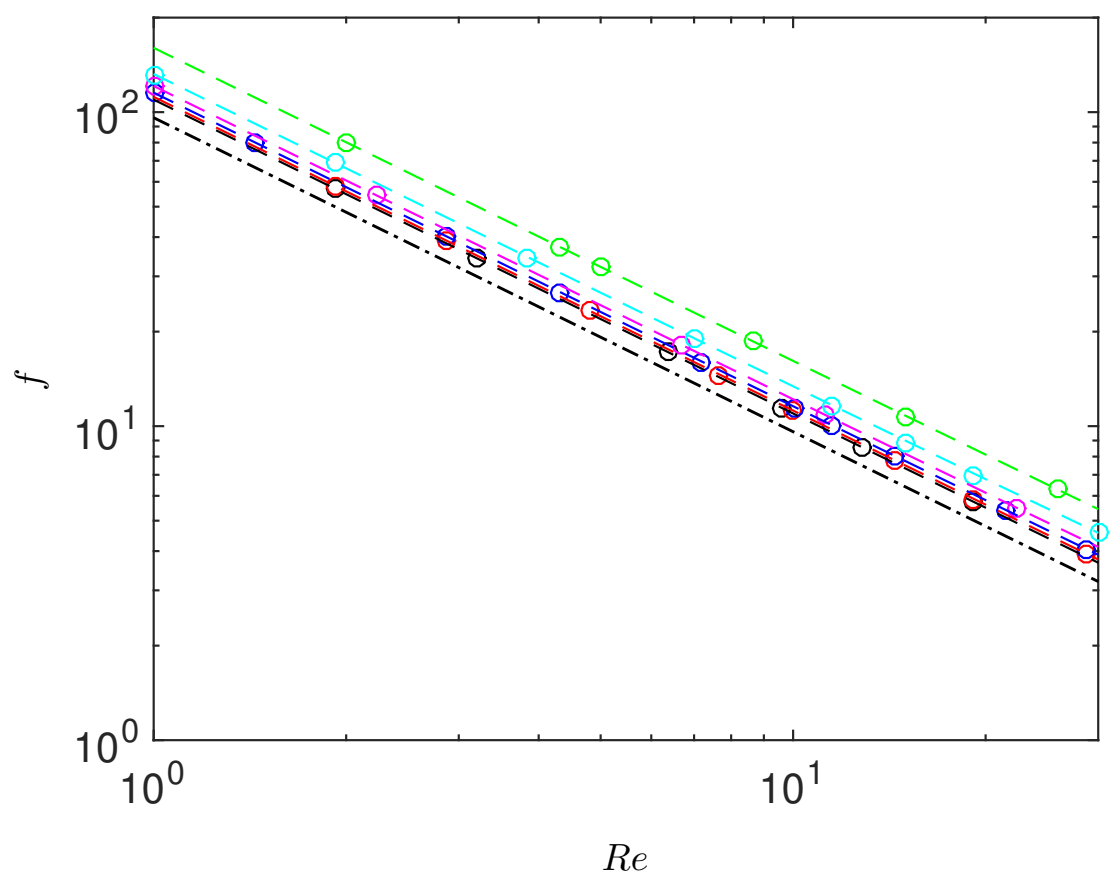

(a)

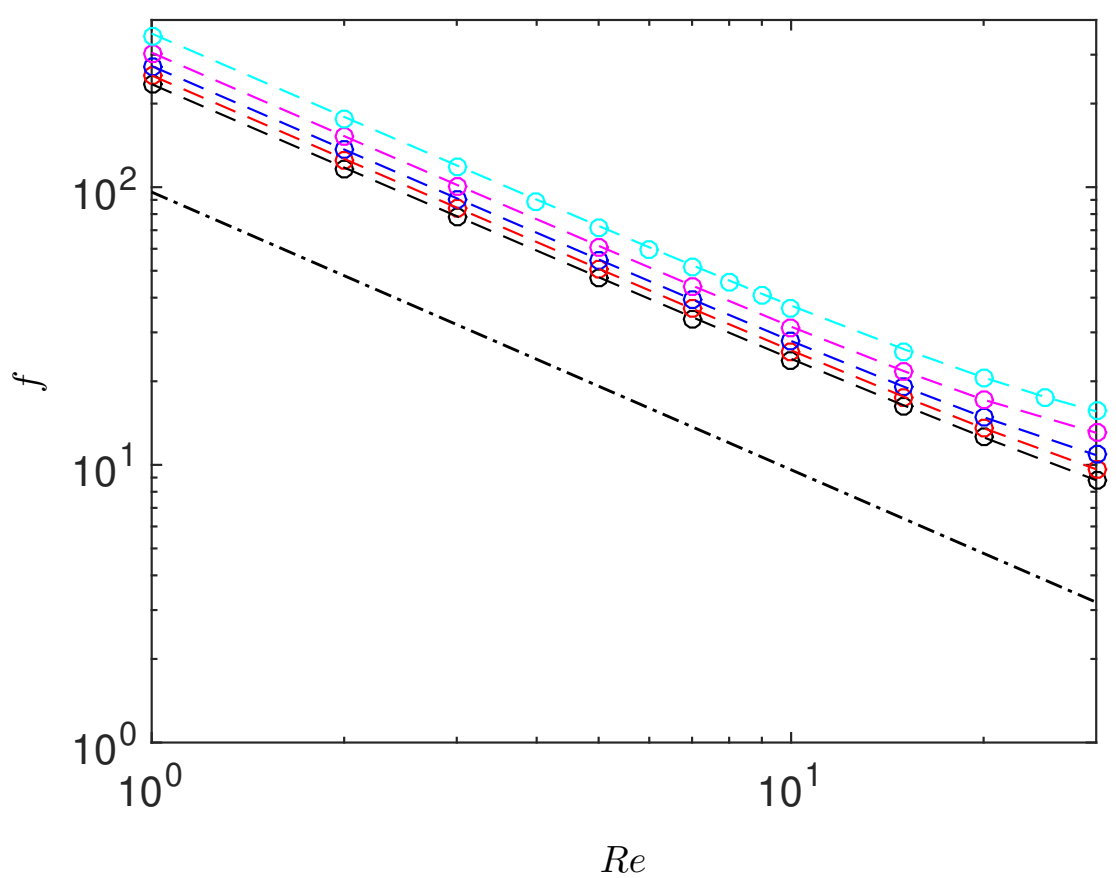

(b)

Fig. 12. Friction factor as a function of the exchanger Reynolds number $R e$ for (a) $\alpha=33^{\circ}$ and (b) $\alpha=90^{\circ}$ with $\circ \varepsilon=0.40$, $\circ \varepsilon=0.50, \circ \varepsilon=0.60, \circ \varepsilon=0.70, \circ \varepsilon=0.80, \circ \varepsilon=0.90 ;-.-$ flow between two parallel plates $f=96 / R e$ 


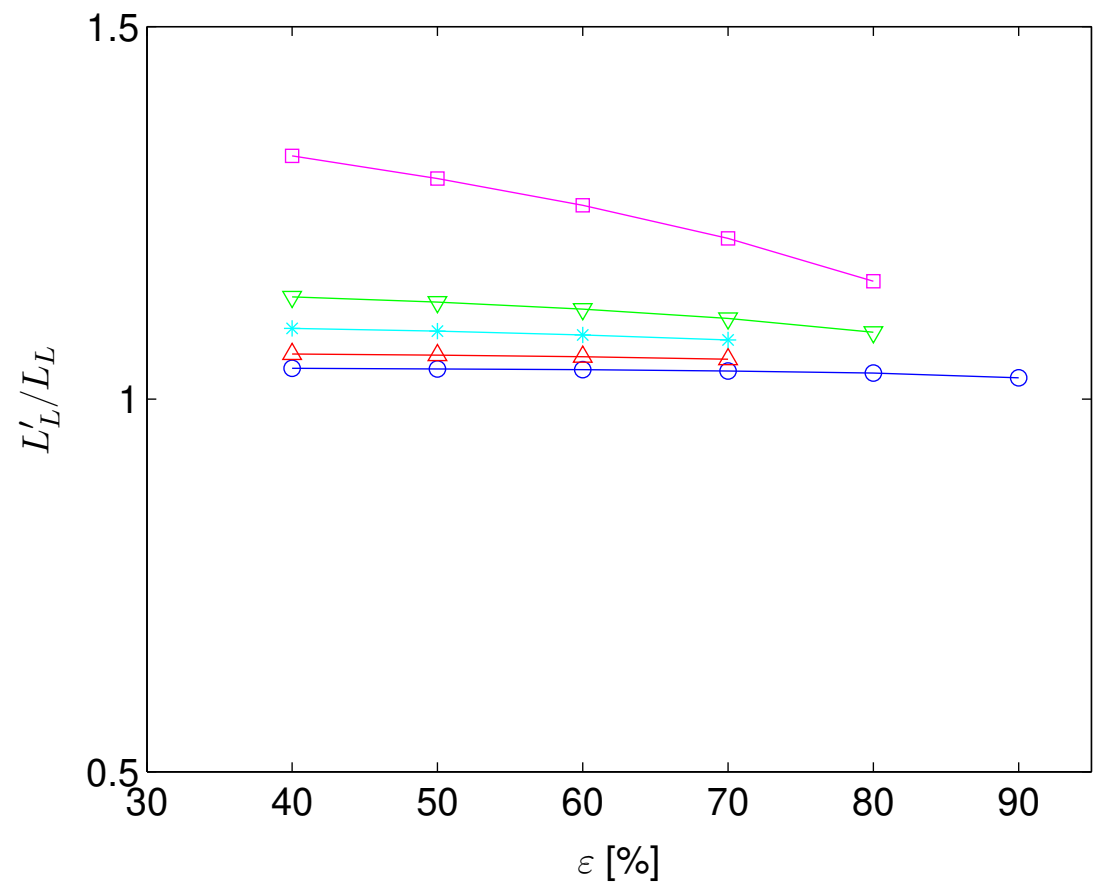

(a)

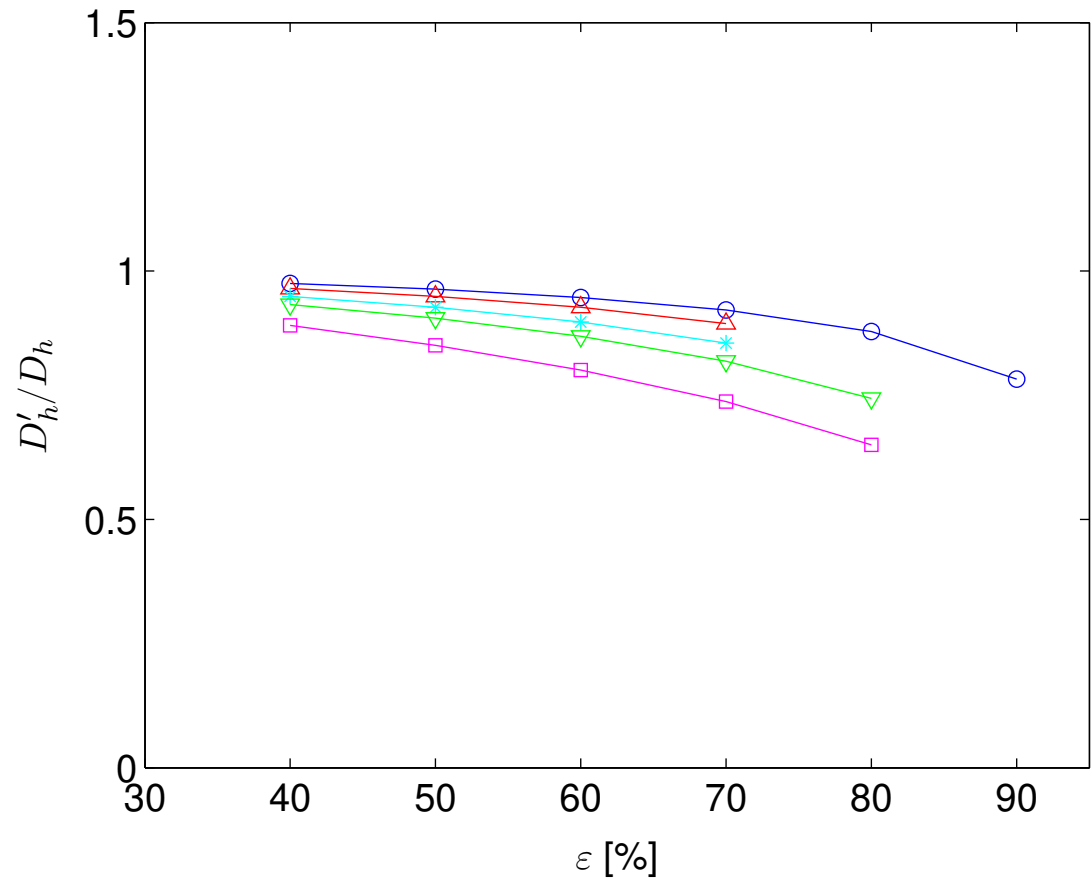

(b)

Fig. 13. (a) Tortuosity and hydraulic diameters ratio as a function of the porosity for : $\circ \alpha=33^{\circ}, \triangle \alpha=40^{\circ},{ }^{*} \alpha=50^{\circ}, \nabla$ $\alpha=60^{\circ}$ et $\square \alpha=90^{\circ}$ 


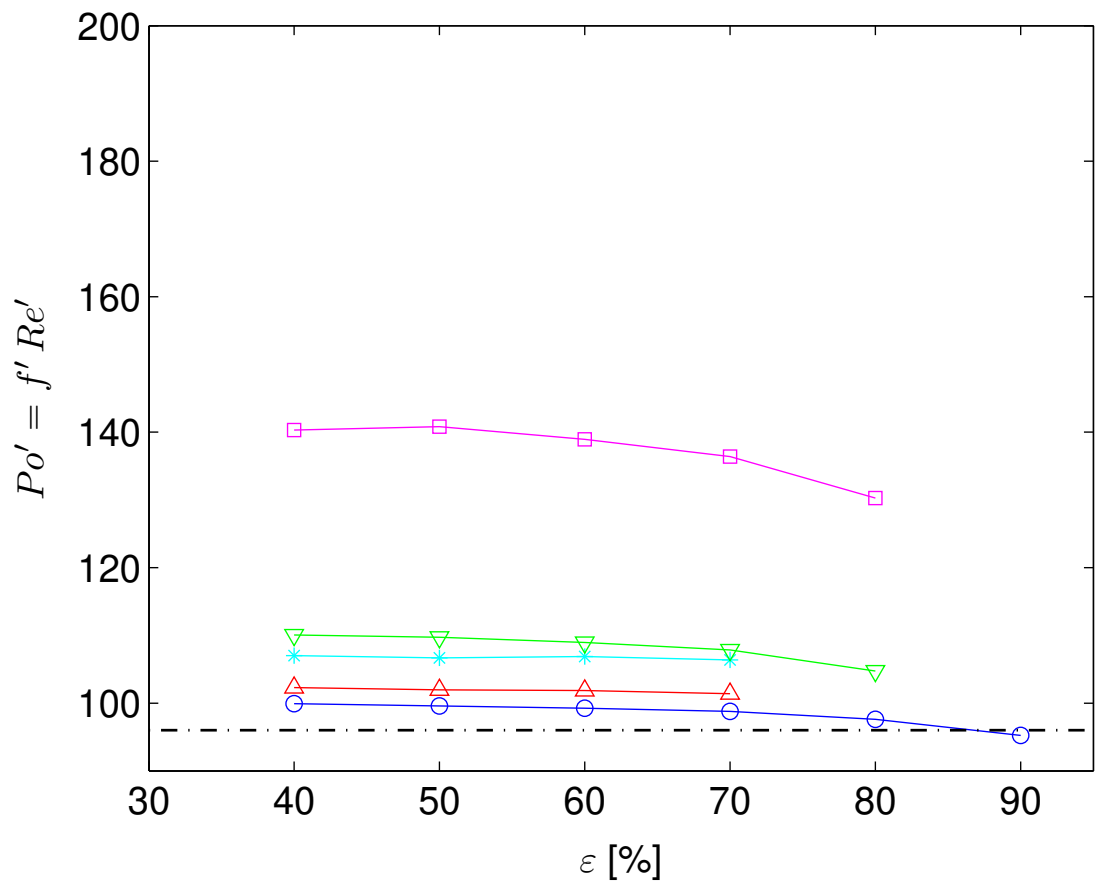

(a)

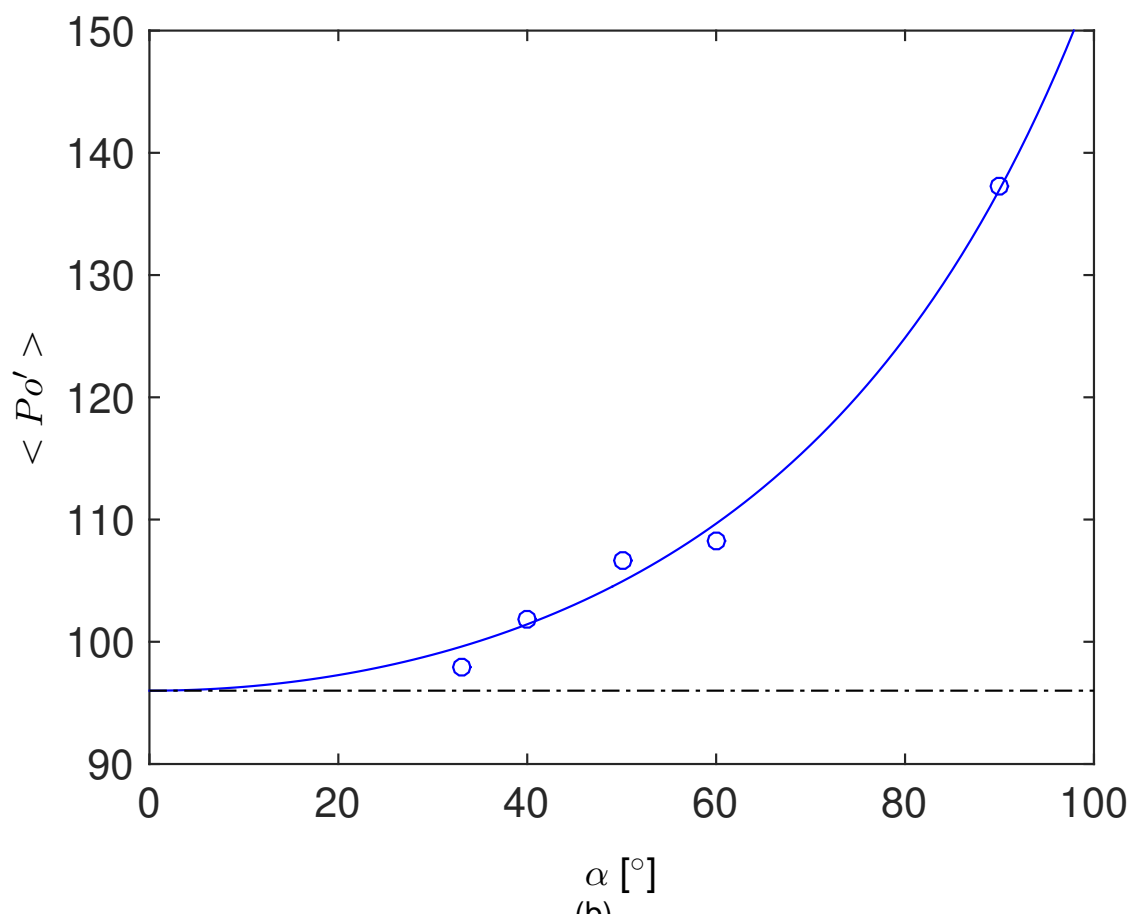

(b)

Fig. 14. Parallel-plates flow analogy (a) Poiseuille number $P o^{\prime}$ as a function of the porosity for: $\circ \alpha=33^{\circ}, \triangle \alpha=40^{\circ}$, * $\alpha=50^{\circ}, \nabla \alpha=60^{\circ}$ et $\square \alpha=90^{\circ}$; -.- flow between two parallel plates $P o^{\prime}=96$; (b) Corresponding average Poiseuille numbers $<P O^{\prime}>$ as a function of the apex angle: 0 numerical simulations and - Eq. 24 


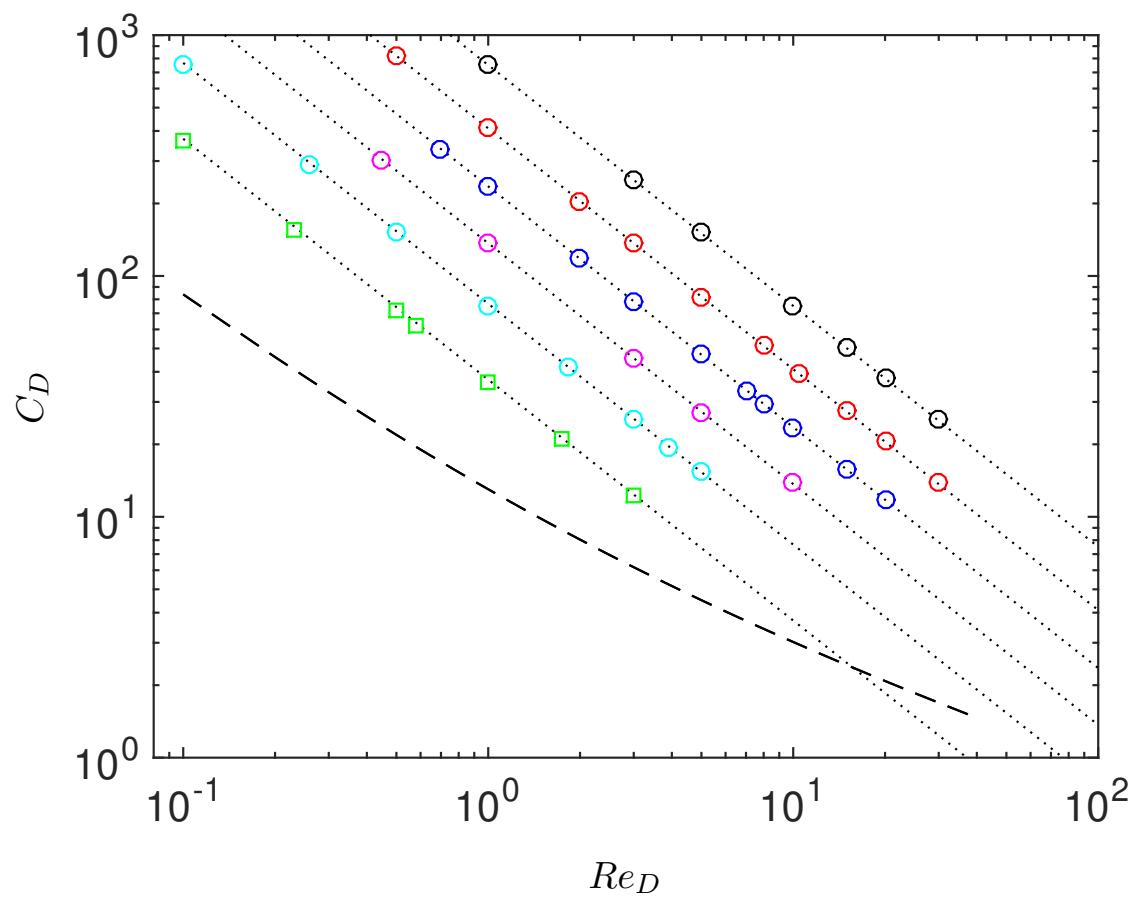

(a)

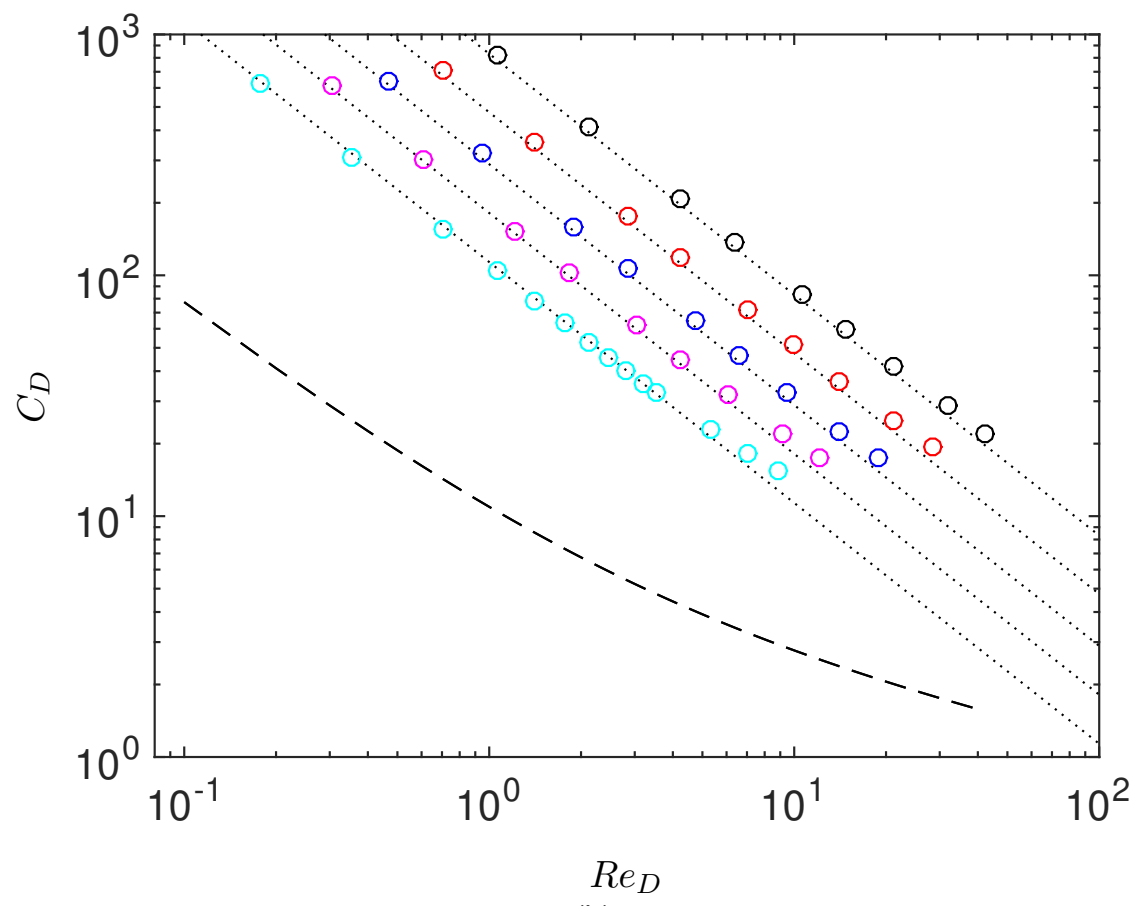

(b)

Fig. 15. Drag coefficient as a function of the cylinder Reynolds number $R e_{D}$ : (a) $\alpha=33^{\circ}$ (b) $\alpha=90^{\circ}$ and $\circ \varepsilon=0.40$, 。 $\varepsilon=0.50, \circ \varepsilon=0.60, \circ \varepsilon=0.70, \circ \varepsilon=0.80, \circ \varepsilon=0.90 ;---C_{D}$ drag coefficient for a single cylinder according to Sochinskii et al. [52]; .... estimation of the drag coefficient using $\left\langle P o^{\prime}\right\rangle$ Eq. 24 and 25 


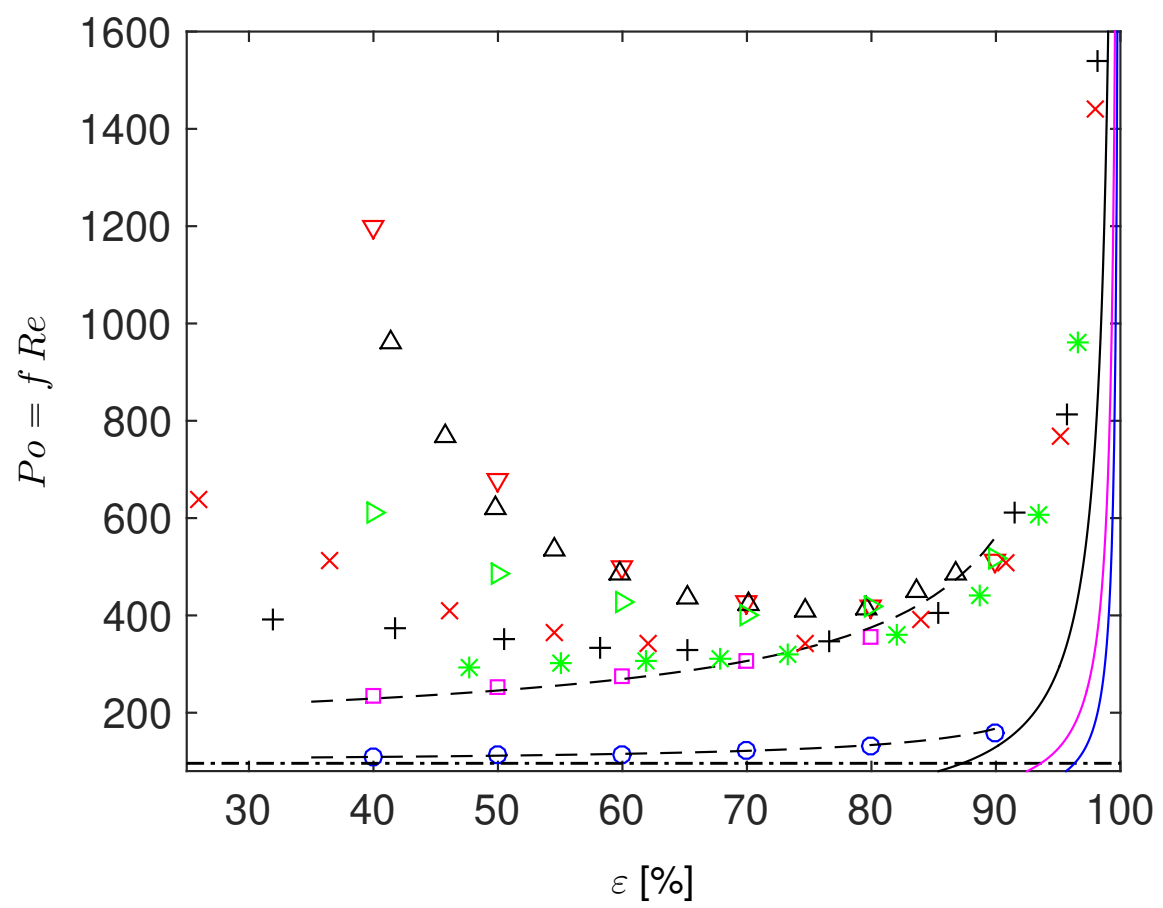

Fig. 16. Poiseuille number comparison to the circular cylinders and spherical particles array cases: square array of circular cylinders according to $\nabla$ numerical simulations of Sangani \& Acrivos [2] and $\triangle$ experiments of Yeom et al. [6]; $\triangleright$ staggered array of circular cylinders according to numerical simulations of Sangani \& Acrivos [2]; * simple cubic array of spheres, + body centered cubic array of spheres, $\times$ face centered cubic array of spheres according to numerical simulations of Sangani \& Acrivos [56]; present numerical simulations with diamond-shaped cylinders arrays for $\circ \alpha=33^{\circ}$ and $\square \alpha=90^{\circ} ;---$ estimation of the Poiseuille number applying the parallel-plates flow analogy $\left(P o=<P o^{\prime}>\left(D_{h} / D_{h}^{\prime}\right)^{2}\left(L_{L}^{\prime} / L_{L}\right)\right.$ and Eq. 24); -.- flow between two parallel plates $P_{O}=96$; asymptotic behaviour for very dilute - spheres arrays and diamond-shaped cylinders arrays with $-\alpha=33^{\circ}$ and $\alpha=90^{\circ}$ (Eq. 27) 


\section{APPENDIX A: LINK BETWEEN FRICTION FACTOR AND DRAG COEFFICIENT}

In a common steady incompressible flow, we recall here the way to link the pressure loss to the drag force exerted on internal surfaces simply by writing the global momentum balance on the calculation domain. The momentum balance in a volume can be written as follow

$$
\rho \iint_{\forall S}(\mathbf{u} \cdot \mathbf{n}) \cdot \mathbf{u}=\iint_{\forall S-S_{f}} p \cdot \mathbf{n}+\iint_{\forall S-S_{f}} \overline{\bar{\tau}} \cdot \mathbf{n}+\mathbf{F}_{S_{f}}
$$

with $\mathbf{n}$ the unit vector normal to the volume faces (outgoing), $\mathbf{S}$ are all the surfaces at the boundary of the volume, $\mathbf{u}$ is the velocity profile at the surfaces. $S_{f}$ are the surface where a drag force is exerted, $\mathbf{F}_{S_{f}}$ is the drag force applied on internal surfaces $S_{f} . p$ and $\overline{\bar{\tau}}$ are respectively the pressure and the viscous constraints. $\forall S$ means that the surface integration is performed on all the boundary surfaces. While $\left(\forall S-S_{f}\right)$ indicates that the surface integration is performed on all the boundary surfaces except the one where we estimate the drag force. This balance corresponds to the classical Euler theorem for a steady flow with the addition of the variation of momentum due to viscous effects.

In the present periodic domain, $S_{f}$ corresponds to the two cylinders surfaces and $\mathbf{F}_{S_{f}}=2 F H$ is twice the drag force applied on one cylinder. The first term of the equation 28 on the LHS corresponds to the variation of the advective flux of the momentum, it is null when the normal unit vector and the velocity are perpendicular to each other such as along wall or symmetry boundary. In addition, the periodic condition on the velocity imposes a zero advective flux of momentum in our calculation domain $\left(\mathbf{u}(x=0)=\mathbf{u}\left(x=L_{L}\right)\right)$. The first term on the RHS corresponds to the variation of the momentum in the flow due to the pressure constraints. Since the pressure is constant at the inlet and outlet of the periodic domain, this integration is equal to $H L_{T}\left(P_{\text {in }}-P_{\text {out }}\right)$. The second term on the RHS corresponds to the momentum variation induced by the viscous constraints linked to velocity gradient. Again because of the periodic condition, the velocity gradients at the inlet and outlet are so close to each over that this term is almost cancelled in practice. As a result, in our geometry the momentum balance can be simplified as 


$$
L_{T}\left(P_{\text {in }}-P_{\text {out }}\right)=2 F
$$

In the literature on flow in periodic domains this result is also found in the following format (see for example $[54,55])$,

$$
\frac{\partial P}{\partial x} V_{t o t a l}=-\sum F
$$

with $\partial P / \partial x=\left(P_{\text {in }}-P_{\text {out }}\right) / L_{L}$ the pressure gradient along the domain, the domain total volume $V_{\text {total }}=L_{L} L_{T}$ and $\sum F$ the sum of forces applied on objects in the calculation domain. Indeed Eq. 29 corresponds to Eq. 30 rewritten as

$$
\frac{L_{T} L_{L}}{D_{h}} \frac{\left(P_{\text {in }}-P_{\text {out }}\right) D_{h}}{0.5 \rho U^{2} L_{L}}=2 \frac{F}{0.5 \rho U^{2}}
$$

By using the expression of the friction factor $f=\left(P_{\text {in }}-P_{\text {out }}\right) D_{h} /\left(0.5 \rho U^{2} L_{L}\right)$ and the drag force $F=0.5 \rho U^{2} a C_{D}$, it is then easy to established the link between the drag coefficient and $\mathrm{f}$ as

$$
\begin{aligned}
\frac{L_{T} L_{L}}{D_{h}} f & =2 a C_{D} \\
C_{D} & =f \frac{L_{T} L_{L}}{D_{h} 2 a}
\end{aligned}
$$




\section{APPENDIX B: POISEUILLE NUMBER OF AN ARRAY OF CIRCULAR CYLINDERS OR SPHER- ICAL PARTICLES}

As reported in the previous appendix, the pressure gradient along the flow is given by

$$
\frac{d P}{d x}=-\frac{\sum\left\|\mathbf{F}_{d}\right\|}{V_{\text {total }}}
$$

Where $\left\|\mathbf{F}_{d}\right\|$ is the drag force magnitude on cylinders or particles in the considered periodic box, while $V_{\text {total }}$ is its total volume.

\section{Poiseuille number for a circular cylinders array}

We consider here a square and a staggered array of cylinders of size $L \times L \times H$ corresponding to a total volume of $V_{\text {total }}=L^{2} H$ with $H>>L$.

The friction coefficient and the Poiseuille number can then be expressed as

$$
\begin{aligned}
f & =\frac{|d P / d x|}{(1 / 2) \rho U^{2}} D_{h}=\frac{\sum\left\|\mathbf{F}_{d}\right\| / H / L^{2}}{(1 / 2) \rho U^{2}} D_{h} \\
P o & =f R e=\frac{\sum\left\|\mathbf{F}_{d}\right\| / H / L^{2}}{(1 / 2) \rho U^{2}} D_{h}\left(\rho D_{h} U\right) / \mu \\
P o & =2\left[\frac{\sum\left\|\mathbf{F}_{d}\right\| / H}{U \mu}\right] \frac{D_{h}^{2}}{L^{2}}
\end{aligned}
$$

The cylinder's diameter can be calculated from the porosity $\varepsilon$ as follow

$$
\begin{aligned}
\varepsilon & =1-n \frac{\pi D^{2} / 4}{V_{\text {total }}}=1-n \frac{\pi D^{2} / 4}{L^{2}} \\
D & =\left[4 L^{2}(1-\varepsilon) /(n \pi)\right]^{1 / 2}
\end{aligned}
$$

with $n=1$ for a square array of circular cylinders $\left(\varepsilon_{\min }=21.5 \%\right)$ and $n=2$ for a staggered 
array of circular cylinders $\left(\varepsilon_{\min }=9.3 \%\right)$. In addition, the corresponding hydraulic diameter is simply $\left(A_{\text {cont }}=n \pi D H\right)$

$$
D_{h}=\frac{4 \varepsilon L^{2}}{n \pi D}
$$

For a periodic box, the sum of drag force per cylinder length applied in the periodic box is the one applied on the $\mathrm{n}$ cylinders $\sum\left\|\mathbf{F}_{d}\right\| / H=n F$ and the Poiseuille expression is

$$
P o=2 n\left[\frac{F}{U \mu}\right] \frac{D_{h}^{2}}{L^{2}}
$$

where the dimensionless drag force $F /(U \mu)$ can be found in the literature from the numerical work of Sangani \& Acrivos [2] (Table 1 and 2 in the article) or from the hybrid model of Yeom et al. [6] constructed on experimental measurements.

\section{Poiseuille number of a spherical particles array}

For a spherical particles array of size $L \times L \times L\left(V_{\text {total }}=L^{3}\right)$, the Poiseuille number can be expressed as

$$
P o=2\left[\frac{\sum\left\|\mathbf{F}_{d}\right\|}{U \mu}\right] \frac{D_{h}^{2}}{L^{3}}
$$

The diameter of the particle can be linked to the porosity as follows

$$
\varepsilon=1-n \frac{\pi D^{3}}{6 L^{3}}
$$


Journal of Fluids Engineering

$$
D=\left(\frac{(1-\varepsilon) 6 L^{3}}{n \pi}\right)^{1 / 3}
$$

with $n=1$ for a simple cubic array $\left(\varepsilon_{\min }=48 \%\right), n=2$ for a body centred cubic array $\left(\varepsilon_{\min }=32 \%\right)$ and $n=4$ for a face centred cubic array $\left(\varepsilon_{\min }=26 \%\right)$. In addition, the corresponding hydraulic diameter is

$$
D_{h}=\frac{4 \varepsilon L^{3}}{n \pi D^{2}}
$$

The sum of drag forces applied in the periodic box is $n$ times the one applied on one particle $\sum\left\|\mathbf{F}_{d}\right\|=n F$ and the Poiseuille expression is

$$
P o=2\left[\frac{n F}{U \mu}\right] \frac{D_{h}^{2}}{L^{3}}=6 n \pi\left[\frac{F}{3 \pi D U \mu}\right] \frac{D D_{h}^{2}}{L^{3}}
$$

where the dimensionless drag force for one particle $F /(3 \pi D U \mu)$ can be found in the literature from the numerical work of Sangani \& Acrivos [56] (Table 3 in the article, 'K converged' values). 